\title{
LXRs regulate features of age-related macular degeneration and may be a potential therapeutic target
}

\author{
Mayur Choudhary, ${ }^{1}$ Ebraheim N. Ismail, ${ }^{2}$ Pei-Li Yao, ${ }^{1}$ Faryan Tayyari,, Roxana A. Radu, ${ }^{3}$ \\ Steven Nusinowitz, ${ }^{3}$ Michael E. Boulton, ${ }^{4}$ Rajendra S. Apte, ${ }^{5}$ Jeffrey W. Ruberti, ${ }^{2}$ James T. Handa, ${ }^{6}$ \\ Peter Tontonoz, ${ }^{7}$ and Goldis Malek ${ }^{1,8}$ \\ 'Duke Eye Center, Department of Ophthalmology, Duke University School of Medicine, Durham, North Carolina, USA. \\ ${ }^{2}$ Department of Bioengineering, Northeastern University, Boston, Massachusetts, USA. ${ }^{3}$ Stein Eye Institute, Department \\ of Ophthalmology, UCLA, Los Angeles, California, USA. ${ }^{4}$ Department of Ophthalmology and Visual Sciences, University of \\ Alabama at Birmingham, Birmingham, Alabama, USA. ${ }^{5}$ Department of Ophthalmology and Visual Sciences, Washington \\ University in Saint Louis School of Medicine, Saint Louis, Missouri, USA. ${ }^{6}$ Wilmer Eye Institute, Johns Hopkins School of \\ Medicine, Baltimore, Maryland, USA. 'Department of Pathology and Laboratory Medicine, UCLA, Los Angeles, California, \\ USA. ${ }^{8}$ Department of Pathology, Duke University School of Medicine, Durham, North Carolina, USA
}

Effective treatments and animal models for the most prevalent neurodegenerative form of blindness in elderly people, called age-related macular degeneration (AMD), are lacking. Genomewide association studies have identified lipid metabolism and inflammation as AMD-associated pathogenic pathways. Given liver X receptors (LXRs), encoded by the nuclear receptor subfamily 1 group $\mathrm{H}$ members 2 and 3 (NR1H3 and NR1H2), are master regulators of these pathways, herein we investigated the role of LXR in human and mouse eyes as a function of age and disease and tested the therapeutic potential of targeting LXR. We identified immunopositive LXR fragments in human extracellular early dry AMD lesions and a decrease in LXR expression within the retinal pigment epithelium (RPE) as a function of age. Aged mice lacking LXR presented with isoformdependent ocular pathologies. Specifically, loss of the Nr1h3 isoform resulted in pathobiologies aligned with AMD, supported by compromised visual function, accumulation of native and oxidized lipids in the outer retina, and upregulation of ocular inflammatory cytokines, while absence of Nr1h2 was associated with ocular lipoidal degeneration. LXR activation not only ameliorated lipid accumulation and oxidant-induced injury in RPE cells but also decreased ocular inflammatory markers and lipid deposition in a mouse model, thereby providing translational support for pursuing LXR-active pharmaceuticals as potential therapies for dry AMD.

Conflict of interest: The authors have declared that no conflicts of interest exist.

Copyright: (ㄷ) 2020, American Society for Clinical Investigation.

Submitted: July 22, 2019

Accepted: December 5, 2019

Published: December 12, 2019.

Reference information: JCI Insight. 2020;5(1):e131928.

https://doi.org/10.1172/jci.

insight.131928.

\section{Introduction}

Dysregulated lipid metabolism and inflammation are key contributors to the development of multiple diseases of aging, including the ocular neurodegenerative disease, age-related macular degeneration (AMD), a leading cause of blindness in the elderly in developed nations, which affects over 130 million people in toto $(1,2)$. A hallmark of the "early" dry clinical subtype of AMD is choriocapillary dropout (3) as well as accumulation of lipid- and protein-rich deposits, known as drusen, below the retinal pigment epithelial (RPE) layer, support cells to the retina $(4,5)$. Although the pathogenesis of early dry AMD remains to be fully understood, an array of risk factors have been identified, implicating disease-associated signaling pathways, most notably, age, along with environmental (e.g., smoking), and genetic factors (e.g., genes associated with inflammatory, complement, and lipid-regulating pathways) (6-9). In spite of the wealth of accepted knowledge regarding potential risk factors, treatment options remain tenuous for patients suffering from the "early" dry form of AMD. Therefore, there is a considerable need not only to identify critical signaling pathways that drive the initiation and progression of AMD but also to develop appropriate animal models that could then be used as a platform to test potential therapies and further discover disease-regulating pathways.

Nuclear receptors are the largest family of transcription factors in the human genome and facilitate the expression of a variety of genes important in maintaining cellular homeostasis during development 
and aging $(10,11)$. Importantly, they play a regulatory role in diseases of aging including Alzheimer's disease $(12,13)$, cardiovascular disease (14), and cancer (15). Given the commonality between pathogenic pathways regulated by nuclear receptors in age-related diseases and AMD, in an attempt to identify candidate receptors that may be important in disease initiation and progression, we previously developed a nuclear receptor atlas of human RPE cells, cells vulnerable in AMD (16). The liver X receptor (LXR; nuclear receptor subfamily 1 group $\mathrm{H}, \mathrm{NR} 1 \mathrm{H}$ ), emerged as a promising target, in light of its role as a regulator of cholesterol homeostasis $(17,18)$ and inflammation $(18), 2$ pathways modulated in AMD (19). Further justification for investigating the role of LXR in the aging eye comes from genomewide association studies, which have found a correlation between single nucleotide polymorphisms in genes involved in cholesterol metabolism/transport and AMD, including member 1 of human transporter subfamily ATP binding cassette transporter $(A B C A 1)$, cholesteryl ester transfer protein, hepatic triglyceride lipase C, and lipoprotein lipase (9, 20, 21), known LXR target genes (22).

To test the hypothesis that the LXR signaling pathway regulates AMD pathology and may serve as a potential target for therapy, we interrogated the role of the LXR pathway in ocular cells vulnerable in AMD. We evaluated the expression of LXR in human RPE cells derived from donor eyes as a function of age, examined the distribution of LXR in human donor eyes as a function of disease, and compared LXR expression throughout compartments of the ocular posterior pole. Activation of the receptor was further evaluated in vitro, under AMD-relevant stressed conditions with pharmacological LXR ligands. The ocular function and phenotype of aged mice lacking the LXR isoforms $\left(\mathrm{Nr}_{1 h 3^{-/-}}, \mathrm{Nr} 1 \mathrm{~h} 2^{-/-}\right.$, and $\left.\mathrm{Nr} 1 \mathrm{~h} 3^{-/-} \mathrm{Nr} 1 \mathrm{~h} 2^{-/-}\right)$were catalogued and the therapeutic potential of activating the LXR signaling pathway on the "AMD" phenotype, including extracellular lipid accumulation and inflammation in the back of the mouse eye, was tested. We report a decrease in NR1H3 expression in human RPE cells as a function of age and accumulation of LXR immunopositive fragments within drusen of dry AMD eyes, reinforcing vulnerability to AMD development through this pathway. We found that LXR-activating ligands are able to improve mitochondrial function and decrease lipid load in RPE cells following injury. Furthermore, we established that the absence of LXR affects the visual function and ocular phenotype of mice differentially, with the absence of the $N r 1 h 3$ isoform resulting in the accumulation of extracellular lipid- and protein-rich deposits, phenotypic features of dry AMD, while the absence of the $N r 1 h 2$ isoform also resulted in non-AMD phenotypes, namely stellate cataract formation and RPE-associated lipoidal degeneration. Detailed analysis of mouse eyes lacking $\mathrm{Nr} / \mathrm{h} 3$ revealed a proinflammatory microenvironment, as evidenced by the accumulation of immune cells in the outer retina and confirmed through analysis of the cytokine profile of the mouse RPE-choroid. Finally, LXR activation was able to reduce AMD-relevant pathogenic phenotypes, including posterior pole ocular inflammation and lipid deposition in mice expressing apoB100, a model for aging and early AMD-associated phenotypes $(23,24)$.

Our studies, collectively, highlight the importance of the NR1H3 signaling pathway in the health of aged RPE cells and present a potentially novel mouse model for the dry AMD phenotype featuring lipid-rich sub-RPE deposits along with accumulation of subretinal immune cells. This model may serve as a platform to test new therapies and identify additional therapeutic targets. Importantly, our results provide translational support for approaches including direct activation of the LXR pathway, which is capable of reducing AMD-associated pathologies and may be a therapeutic target for not only the early dry form of AMD but also other ocular and nonocular diseases characterized by either abnormal lipid deposition or tissue immune cell infiltration.

\section{Results}

$L X R$ expression in human RPE cells decreases as a function of age. Given the critical role of advanced age in the development of AMD, we measured the relative expression of NR1H3 (LXRA) and NR1H2 (LXRB) in human RPE cells isolated from donor tissue as a function of age (Figure 1, Supplemental Figure 1, and Supplemental Table 1; supplemental material available online with this article; https://doi.org/10.1172/ jci.insight.131928DS1). A significant negative correlation was found between relative NR1H3 and NR1H2 expression levels and age (Figure 1A and Supplemental Figure 1A). This negative correlation remained significant for NR1H3 expression, not NR1H2, in the "at risk for AMD" age range of 60-94 years, suggesting a steady decline in NR1H3 but a plateau in NR1H2 expression in human RPE cells (Figure 1B and Supplemental Figure 1B). In a smaller cohort of RPE tissue from middle- to advanced-age donors, 
A

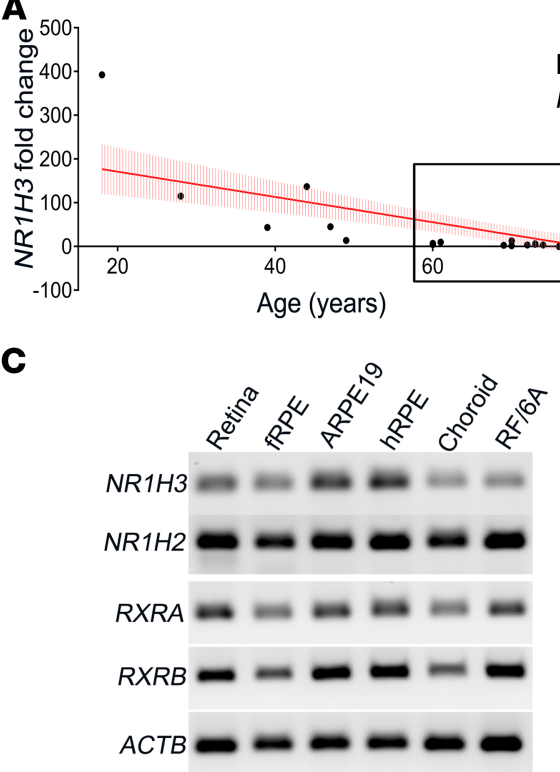

B
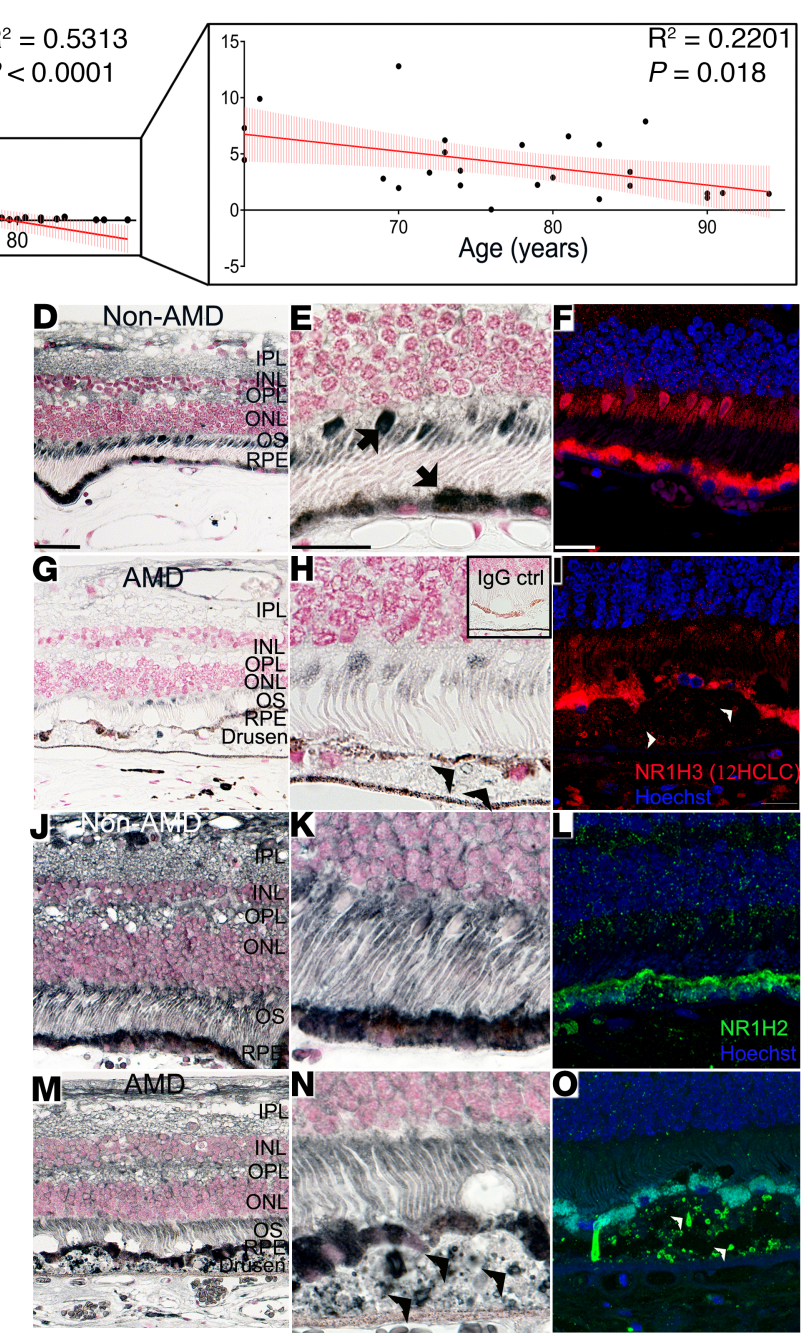

Figure 1. NR1H3 expression in RPE declines with age. (A) Expression of NR1H3 in RPE cells isolated postmortem from donors, 18-94 years old; fold change relative to ARPE19; $n=31$. Pearson's $R^{2}$ was calculated, $P<0.0001$. (B) Expression of NR1H3 in RPE cells isolated postmortem from donors, $60-94$ years old; fold change relative to ARPE19, $n=25$. Pearson's $R^{2}$ was calculated; $P=0.018$. (C) Agarose gel image of genomic DNA amplification products of NR1H3, $N R 1 H 2$, and their obligate binding partners retinoid $X$ receptors $A$ and $B(R X R A$ and $R X R B$ ) in retina, freshly isolated human RPE cells (fRPE), ARPE19 cells, primary human RPE cells (hRPE), human choroid, and RF/6A cells. $\beta$-Actin (ACTB) was used a loading control. Representative images of NR1H3 (12HCLC) immunohistochemical (IHC) staining (dark gray) in retina/RPE/choroid complex from non-AMD ( $\mathbf{D}$ and $\mathbf{E}$; arrows point to a cone and RPE cells) and AMD (G and $\mathbf{H}$ ) donors. Arrowheads point to dark gray NR1H3 staining within drusen. Inset: IgG control. Scale bars: $50 \mu \mathrm{m}$ (D), $25 \mu \mathrm{m}$ (E). (F and I) NR1H3 immunofluorescence (IF) staining in non-AMD and AMD donors. Arrowheads point to NR1H3 staining, red. Scale bar: $25 \mu \mathrm{m}$ (F). NR1H2 IHC staining in retina/RPE/ choroid complex from non-AMD ( $\mathbf{J}$ and $\mathbf{K}$ ) and AMD ( $\mathbf{M}$ and $\mathbf{N}$ ) donors (arrowheads point to dark gray NR1H2 staining within drusen). ( $\mathbf{L}$ and $\mathbf{O})$ NR1H2 IF staining in non-AMD and AMD donors (arrowheads point to NR1H2 staining within drusen, green). Total number of eyes probed with antibodies ( $n=2-10$ from age-matched non-AMD and AMD donors, respectively). IPL, inner plexiform layer; INL, inner nuclear layer; OPL, outer plexiform layer; ONL, outer nuclear layer; OS, outer segments; RPE, retinal pigment epithelial cells.

expression of LXR target genes $A B C A 1(n=12)$ and of apolipoprotein $\mathrm{E}(A P O E)(n=15)$ were found to increase with age (Supplemental Figure 2, A and B). The purity of freshly isolated RPE cells from human donors was assessed by measuring the expression of bestrophin-1 (BEST1) and RPE-specific protein 65/ retinoid isomerohydrolase RPE65 (RPE65) in freshly isolated RPE, retina, and choroid from corresponding donors. RPE samples ( $n=23$ ) were found to have a $2.13 \times 10^{2}$-fold to $4.52 \times 10^{5}$-fold higher expression of BEST1 and $4.98 \times 10^{2}$-fold to $2.15 \times 10^{4}$-fold higher expression of RPE65, relative to ARPE19 cells. Additionally, the expression levels of BEST1 and RPE65 in retina and choroid were negligible (0.01- to 0.3-fold change for BEST1 and 0.009- to 0.2-fold change for RPE65) as compared with the RPE, reflecting the purity of the samples. Importantly, all the components of the LXR pathway were expressed in freshly isolated tissue and cultured cells (Figure 1C). We next evaluated the localization of NR1H3 and NR1H2 


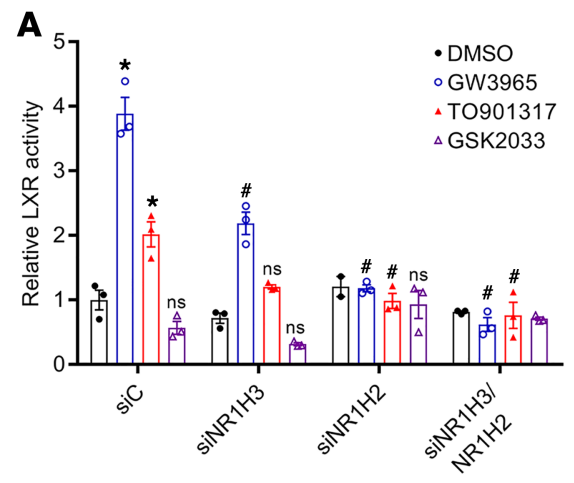

D

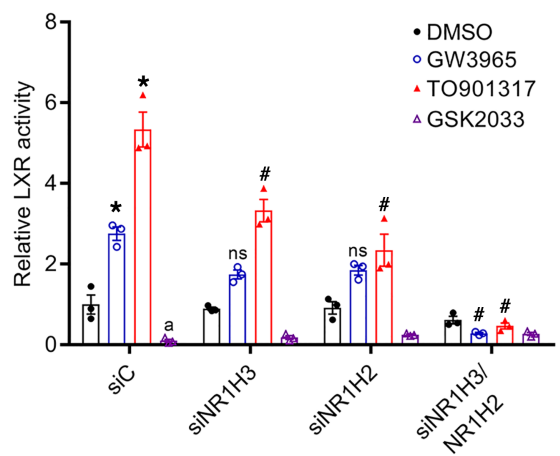

B

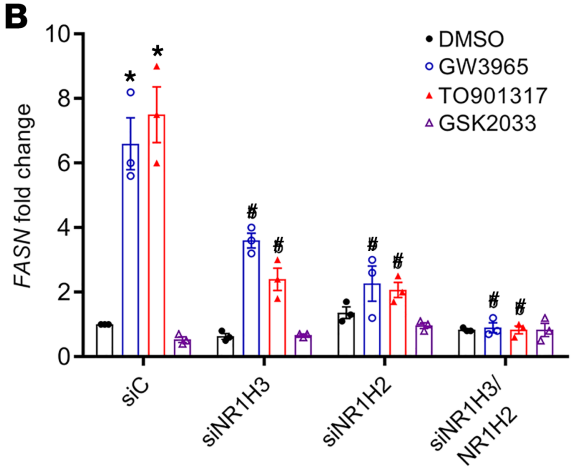

$\mathbf{E}$

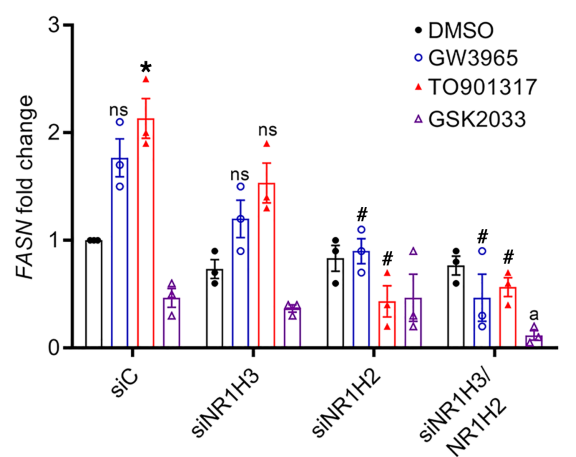

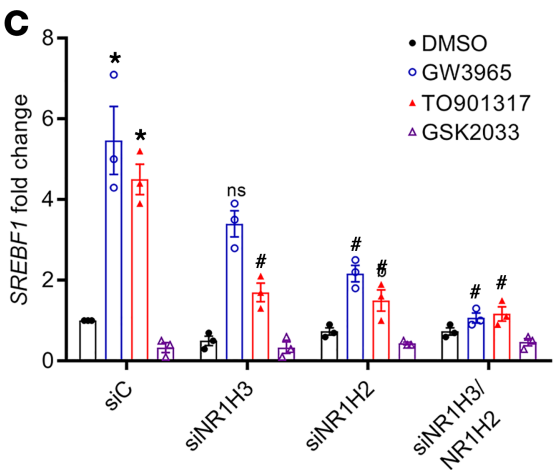

$\mathbf{F}$

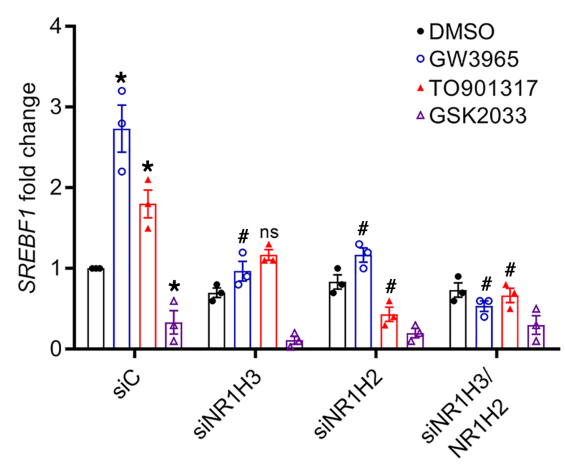

Figure 2. The LXR pathway is biologically active in AMD-vulnerable cells. (A) LXR activity in hRPE cells transfected with DR-4 luciferase reporter and siC (control, nontargeting siRNA), siNR1H3, siNR1H2, or siNR1H3/NR1H2 and treated with LXR agonists GW3965 and TO901317 or antagonist GSK2033. DMSO was used as a vehicle control. $n=3$. Mean \pm SEM shown. ${ }^{*} P<0.05$ relative to siC DMSO; ${ }^{*} P<0.05$ relative to drug siC-treated cells. Two-way ANOVA and Tukey's multiple comparisons. Expression of LXR target genes fatty acid synthase (FASN) (B) and sterol regulatory element-binding protein 1 (SREBF1) (C) in hRPE cells in response to treatment with LXR agonists GW3965 and T0901317 and antagonist GSK2033. $n=3$. Mean \pm SEM. ${ }^{*} P<0.05$ relative to siC DMSO; $P<$ 0.05 relative to drug siC-treated cells, 2-way ANOVA and Tukey's multiple comparisons. (D) LXR activity in CECs transfected with DR-4 luciferase reporter and $\mathrm{siC}$, siNR1H2, siNR1H2, or siNR1H3/NR1H2 cells and treated with LXR agonists CW3965 and T0901317 or antagonist GSK2033. DMSO was used as a vehicle control. $n=3$. Mean \pm SEM. ${ }^{*} P<0.05$ relative to siC DMSO; ${ }^{*} P<0.05$ relative to drug siC-treated cells. Two-way ANOVA and Tukey's multiple comparisons. Expression of LXR target genes FASN (E) and SREBF1 (F) in CECs in response to treatment with LXR agonists GW3965 and TO901317 and antagonist GSK2033. $n=3$. Mean \pm SEM. ${ }^{*} P<0.05$ relative to siC DMSO; ${ }^{P} P<0.05$ relative to drug siC-treated cells, 2 -way ANOVA and Tukey's multiple comparisons.

protein in retinal cross sections from AMD and age-matched unaffected "normal" donor eyes via IHC. In sections from unaffected donors, NR1H3 immunoreactivity was observed in RPE cells and select cones (Figure 1, D-F), while NR1H2 immunoreactivity was also present throughout the inner retina (Figure 1, J-L). In AMD donor sections, NR1H3 immunoreactivity was less evident in the RPE cell layers (Figure 1, G-I) but still present, though at lower levels, and NR1H3-immunopositive fragments were seen in large drusen in 6 out of 9 donor tissues (Figure 1 and Supplemental Figure 3), in addition to cone cells, as shown by red/green opsin staining (Supplemental Figure 4). This staining pattern was supported using a different primary antibody against NR1H3 (Supplemental Figure 4). NR1H2 immunoreactivity in drusen was also abundant in addition to diffuse staining within the neural retina of AMD eyes, reflecting the ubiquitous nature of this isoform (Figure 1, M-O).

The LXR pathway is biologically active in $A M D$-vulnerable cells. NR1H3 and NR1H2 activities were examined in hRPE cells and the macaque-derived RF/6A choroidal endothelial cell (CEC) lines. Receptor transcriptional activity was assessed by measuring the binding of the receptor-ligand complex to the gene response element using a luciferase reporter assay. Ligand activation by GW3965 and TO901317, 2 nonsteroidal LXR-active pharmaceuticals (Supplemental Table 3), significantly increased LXR promoter activity in hRPE (GW3965, 4-fold; TO901317, 2-fold; Figure 2A) and CEC (GW3965, 2.5-fold; TO901317, 5-fold; Figure 2D) lines. This induction was reversed following siRNA-induced knockdown of NR1H3 and NR1H2 expression. Conversely, LXR antagonist (GSK2033) treatment did not result in promoter binding. Ligand activation by GW3965 and TO901317 significantly induced expression of LXR target genes, FASN and SREBF1 in both hRPE (from a 93-year-old female; Figure 2, B and C) and CEC (Figure 2, E and F) lines. Similar induction patterns of LXR target genes in response to agonists were observed in 2 additional primary RPE cell lines (from a 15- and 

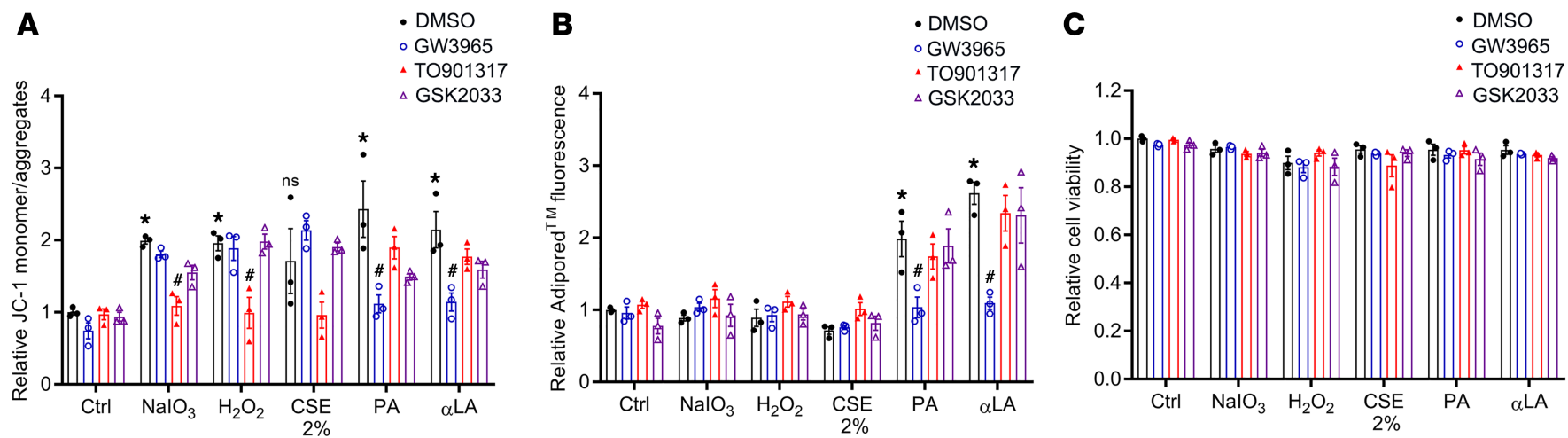

Figure 3. LXR activation differentially ameliorates oxidant injury and lipid overload in a ligand-dependent manner. Human primary RPE cells from a 93-year-old donor were pretreated with LXR agonists or antagonists followed by oxidant injury or lipid overload and the (A) effect of LXR activation on the mitochondrial membrane potential was measured by exposure to the JC-1 dye, in which the ratio of JC-1 monomers/aggregates was used as a measure of mitochondrial injury. (B) Intercellular lipid accumulation was quantified in AdipoRed assays. (C) Cell viability was measured using CellTiter-Blue. Drugs used included the LXR agonists CW3965 and T0901317 and antagonist GSK2033. DMSO and ethanol were used as vehicle controls. $n=3$. Mean \pm SEM shown. ${ }^{*} P<0.05$ relative to vehicle control; ${ }^{\#} P<0.05$ relative to drug control, 2 -way ANOVA and Tukey's multiple comparisons.

60-year-old). Collectively, these results demonstrate that components of the LXR pathway are expressed, and LXR activity and target gene expression can be modulated through pharmacological drugs and siRNA, respectively, in ocular cells vulnerable to AMD. Furthermore, variation in the magnitude of LXR induction and expression illustrates the differential nature and cell-specific effects of these ligands.

LXR activation differentially ameliorates oxidant injury and lipid overload in a ligand-dependent manner in the eye. The retina is susceptible to oxidant injury culminating from inadequate neutralization of generated reactive oxygen species (ROS). This high ROS load is a consequence of the retina's daily exposure to visible light, its high oxygen consumption, the daily phagocytosis of photoreceptor outer segments, as well as its polyunsaturated fatty acid-rich environment (25). Human RPE cells were exposed to agents simulating oxidant injury (i.e., $\mathrm{NaIO}_{3}, \mathrm{H}_{2} \mathrm{O}_{2}$, and cigarette smoke extract, CSE), and lipid overload (i.e., palmitic acid, PA; and $\alpha$-linolenic acid, $\alpha$-LA; Supplemental Table 3). The effects of LXR modulation in response to oxidant- and lipid-induced damage to the RPE cells were determined by evaluating the activity assay, mitochondrial membrane potential using the JC-1 assay, lipid buildup in the cells by AdipoRed assay, and cell viability by CellTiter-Blue assay. We found that the treatment with oxidant injury and lipids did not directly induce LXR activity in RPE cells (Supplemental Figure 5). A 24-hour pretreatment of RPE cells with the LXR ligand TO901317 was able to prevent the compromise to the mitochondrial membrane potential in $\mathrm{NaIO}_{3}$-treated, $\mathrm{H}_{2} \mathrm{O}_{2}$-treated, and CSE-treated cells, whereas GW3965 pretreatment was able to block mitochondrial injury in lipid-treated cells (Figure 3A and Supplemental Figure 6). LXR antagonist GSK2033 pretreatment had no appreciable effect on the mitochondrial membrane potential. Additionally, we measured the neutral lipid content in RPE cells in response to oxidant injury and lipid overload and examined whether LXR pathway modulation could prevent the accumulation of neutral lipids in RPE cells. Lipid treatments (PA and $\alpha$-LA) induced a buildup of neutral lipids within cells, which was ameliorated by pretreatment with GW3965 (Figure 3B). The agonist TO901317, and antagonist GSK2033, did not affect lipid accumulation in RPE cells in vitro. Finally, cell viability assessments confirmed that the drug pretreatments and injury treatments were sublethal for the course of the assay (Figure 3C). These results revealed the therapeutic potential of activating the LXR pathway to avert oxidant injury and lipid accumulation in RPE cells. Importantly, the 2 LXR agonists tested appear to work via distinct pathways, as evident by their selective action in response to diverse injury agents.

Loss of Nr1h3 in vivo compromises visual function. Given the role of LXR in regulating lipid metabolism and inflammation, the 2 pathways associated with development of AMD, and our observation that LXR expression in human RPE cells decreases with age, we examined the visual function of aged LXR-knockout mice, after confirming that the absence of LXR globally also affects LXR gene expression locally in the RPE-choroid (Supplemental Figure 7). Visual function was assessed by recording electroretinograms (ERGs) from 10- to 14-month-old $\mathrm{Nr}_{1 h 3^{-/-}}(n=11), \mathrm{Nr}_{\mathrm{rh}} 2^{-/-}(n=11), \mathrm{Nr}_{\mathrm{H}} 3^{-/-} \mathrm{Nr} 1 \mathrm{~h} 2^{-/-}(n=2)$, and $\mathrm{Nr} 1 \mathrm{~h} 3^{+/+}$ $N r 1 h 2^{+/+}(W T, n=15)$ controls. Evaluation of the ERG recordings revealed a decline in scotopic a-wave 
A

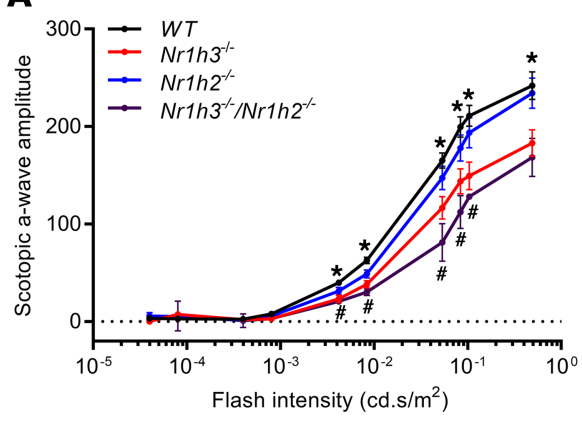

B

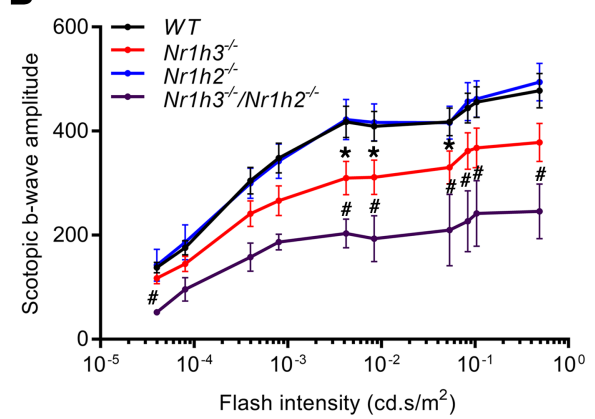

C

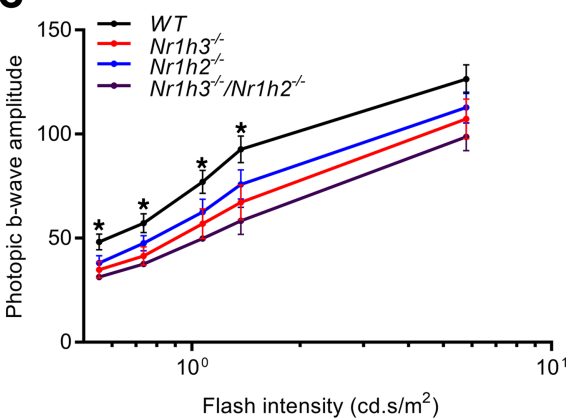

D

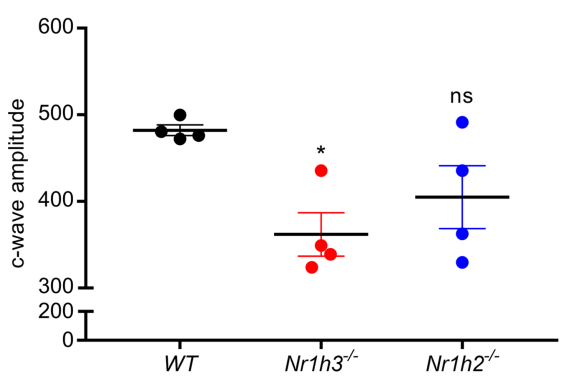

E

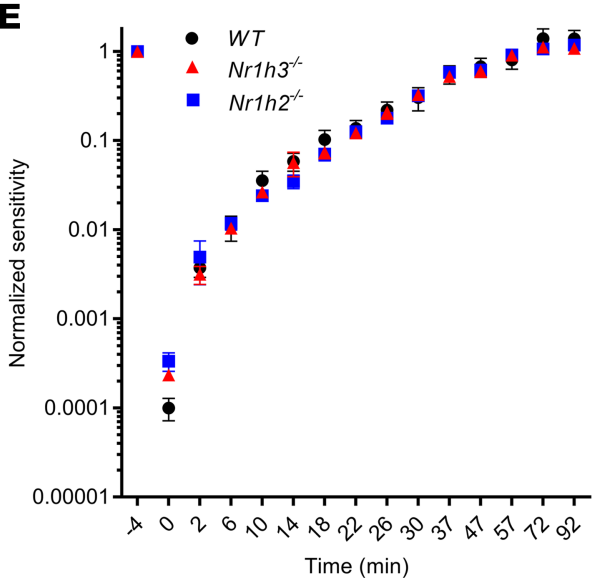

$\mathbf{F}$

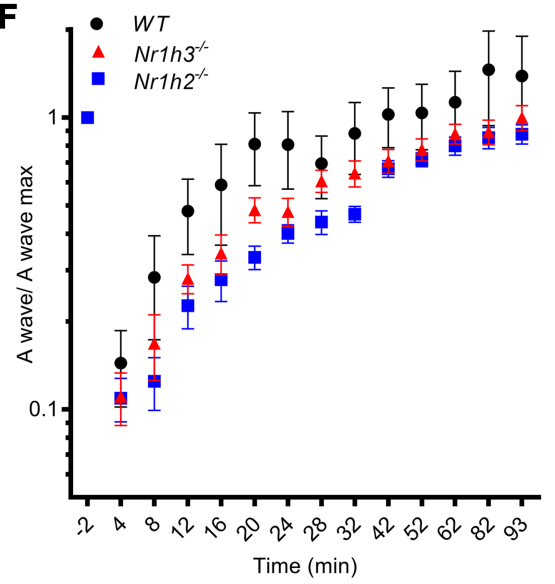

Figure 4. Loss of $\mathrm{Nr} 1 \mathrm{~h} 3$ in vivo compromises visual function. Averaged ERG responses from 10 - to 14-month-old dark-adapted $\mathrm{WT}, \mathrm{Nr} / \mathrm{h} 3^{-/-}, \mathrm{Nr} 1 \mathrm{~h} 2^{-/-}$, and $\mathrm{NrTh}^{-/-} \mathrm{NrTh}^{-1-}$ mice. Plots of (A) scotopic a-wave amplitudes, (B) scotopic b-wave amplitudes, and (C) photopic a-wave amplitudes as a

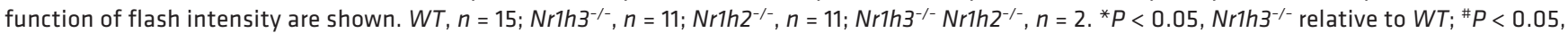
$N r 1 h 2^{-1-}$ relative to $W T$. Mean \pm SEM. Multiple $t$ tests. (D) C-wave amplitudes. $n=4$, Mean \pm SEM shown. ${ }^{*} P<0.05$; ns, not significant; 1 -way ANOVA, Dunnett's multiple-comparisons test. Dark adaptation ERG was performed and results were reported as (E) sensitivity as a function of time (WT: $n=$ 4, $\mathrm{Nr}_{\mathrm{h} 3^{--}}: n=4, \mathrm{Nr}_{\mathrm{h} 2^{-1}:}: n=4$; mean \pm SEM shown) and (F) ratio of a-wave amplitude (a wave)/prebleach a-wave amplitude (A wave max). WT, $n=$

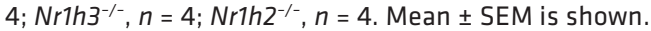

(approximately 41\%), scotopic b-wave (approximately 26\%), and photopic b-wave amplitudes (approximately $28 \%$ ) of $\mathrm{Nr}_{1 \mathrm{~h} 3^{-1-}}$ mice compared with $\mathrm{WT}$ controls (Figure 4, A-C), indicating that in the absence of $N r 1 h 3$, visual function may be compromised at several levels, including the photoreceptors and retinal cells postsynaptic to photoreceptors. We also evaluated the visual function of $\mathrm{Nr}_{\mathrm{rh}} \mathrm{H}^{--}$mice as a function of age and observed an age-related decline in scotopic a-wave and b-wave amplitudes (4- to 5-month-olds compared with 10- to 12-month-olds; Supplemental Figure 8), supporting a role for $\mathrm{Nr} 1 \mathrm{~h} 3$ in the maintenance of retinal cell health. Upon further examination, gene-specific differences in the localization of retinal cell markers for photoreceptors (Supplemental Figure 9, A and B) and Müller and bipolar cells (Supplemental Figure 9, C and D) were not seen, and no differences in retinal layer thicknesses were observed (Supplemental Figure 10). However, a significant decline (24.9\%) in the c-wave amplitude in $\mathrm{Nr} 1 \mathrm{~h} 3^{-1-}$ mice compared with $W T$ controls (Figure 4D) was observed, reflecting the contribution of the RPE to the visual function decline observed in these mice (26). Although $\mathrm{Nr}_{\mathrm{h}} \mathrm{2}^{-/-}$mice did not display any gene-specific differences in visual function, the $N r 1 h 3^{-1-} N r 1 h 2^{-1-}$ mice followed a similar trend to the $N r 1 h 3^{-1-}$ mice (Figure 4). Finally, no significant isoform-specific differences were seen in dark adaptation and a-wave flash sensitivity. Some $L X R$-dependent changes in the recovery kinetics for a-wave amplitude were seen but only at early time points (Figure 4, E and F).

Isoform-specific ocular pathologies indicate absence of $L X R \alpha$ is associated with an early dry AMD phenotype. In vivo imaging allowed examination of the morphology of the fundus and OCT images from aged $\mathrm{Nr} 1 \mathrm{~h} 3^{-/-}$,

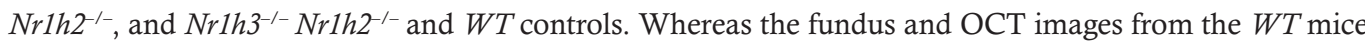
were not remarkable (Figure 5, A and B), the $\mathrm{Nr}_{\mathrm{H}} \mathrm{3}^{-1-}$ mice exhibited hypopigmented regions in the fundus (Figure 5, C and D, and Supplemental Figure 11, A-H). Similarly, $N r 1 h 3^{--}-N r 1 h 2^{-/-}$mouse fundus images presented with pigmentary changes in the fundus (Figure $5, \mathrm{G}$ and $\mathrm{H}$ ). $\mathrm{Nr} 1 \mathrm{~h} 2^{-1-}$ mice, in addition to retinal 

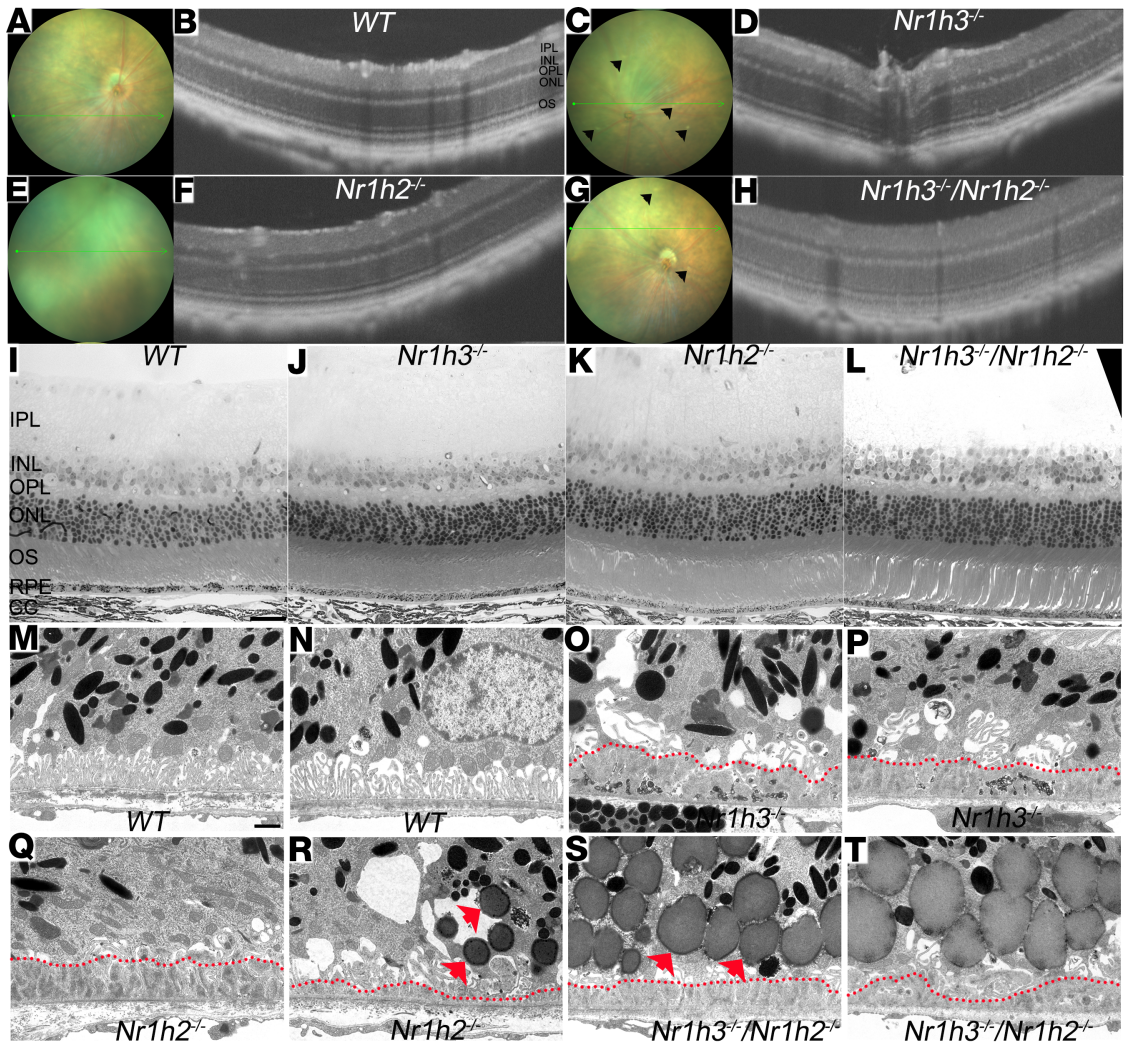

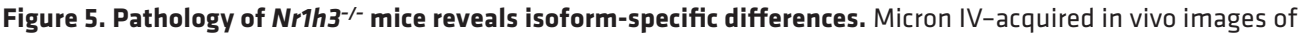

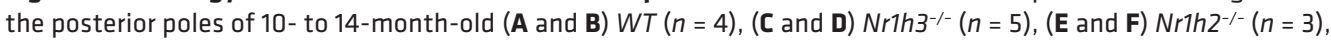
and $(\mathbf{G}$ and $\mathbf{H}) \mathrm{Nr} \mathrm{h}^{-/-} \mathrm{Nr}^{-h_{2} 2^{--}(n=4)}$ mice. Black arrowheads point to hyporeflective spots in the fundus. Toluidine

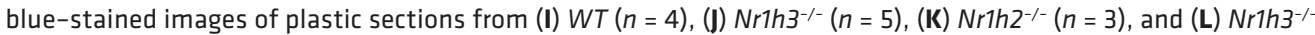
$\mathrm{NrTh}^{-1-}(n=4)$ mice showed normal retinal morphology and no gene-specific differences at the level of the inner retina. Scale bar: $20 \mu \mathrm{M}$ (I). Conventional electron micrographs of RPE/Bruch's membrane/choroidal junction in ( $\mathbf{M}$ and $\mathbf{N}$ ) WT mice display normal RPE morphology, while ( $\mathbf{O}$ and $\mathbf{P}) \mathrm{Nr}_{\mathrm{h}} \mathrm{h}^{-/-}$mice illustrate continuous sub-RPE

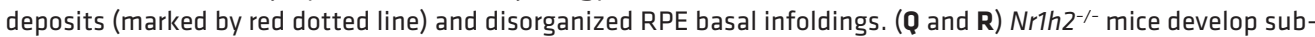
RPE deposits (marked by red dotted line) along with large lipid droplets (red arrowheads) within the RPE, while (S and T) $\mathrm{Nr}_{1} \mathrm{~h}^{-/-} \mathrm{Nr}_{\mathrm{h}} \mathrm{2}^{-/-}$mice display a more severe form of both pathologies. Sub-RPE deposits, red dotted line; lipid droplets, red arrowheads. CTEM scale bar: $1 \mu \mathrm{m}$. CC, choriocapillaris; OS, outer segments; ONL, outer nuclear layer; $\mathrm{OPL}$, outer plexiform layer; INL, inner nuclear layer; IPL, inner plexiform layer.

changes, developed stellate cataracts (Figure 5, E and F, and Supplemental Figure 11, I-L), resulting in cloudy fundus images. The overall architecture of the inner retinas of LXR-null mice was evaluated ex vivo in cross sections, and no gene-specific differences in the overall architecture and thickness of the inner retinal layers were found $\left(N r 1 h 3^{-/-}, n=5 ; N_{r} 1 h 2^{-/-}, n=3 ; N_{r} 1 h 3^{-/-} N_{r} 1 h 2^{-/-}, n=4 ;\right.$ and $W T, n=4 ;$ Figure 5 , I-L, and Supplemental Figure 10). High-magnification evaluation of the outer retina of LXR mice using conventional transmission electron microscopy (cTEM) allowed for detailed analysis of the ultrastructure of the RPE-choroid complex. Although eyes from WT mice exhibited normal RPE-choroid morphology with organized basal infoldings and apical processes of the RPE (Figure 5, M and N), in $\mathrm{Nr}_{1 h 3^{--}}$mice, continuous sub-RPE deposits were observed below disrupted basal infoldings, seen as amorphous, electron-dense material, interspersed with "streamers," a phenotype typical of early dry AMD (over $40 \%$ of the length of Bruch's membrane, BrM; Figure 5, O and P, and Supplemental Figure 12). The RPE phenotype of $\mathrm{Nr}_{\mathrm{h}} \mathrm{h}^{-/-}$mice had distinct pathologies, including accumulation of large lipid droplets within the RPE, reminiscent of lipoidal degeneration, which is not a characteristic of AMD (Figure 5, Q and R, and refs. 27, 28). Predictably, $N r 1 h 3^{-/-} N r 1 h 2^{-/-}$mice displayed both phenotypes, large lipid droplets within the RPE in combination with accumulation of extracellular electron-dense sub-RPE deposits (Figure 5, S and T). Based on these morphological findings we conclude that in the eye the $\alpha$-isoform of LXR regulates extracellular lipid deposition, an early phenotypic feature of dry AMD, while the $\beta$-isoform is responsible for intracellular lipid accumulation as well as stellate cataract, an ocular pathology of the anterior segment. 

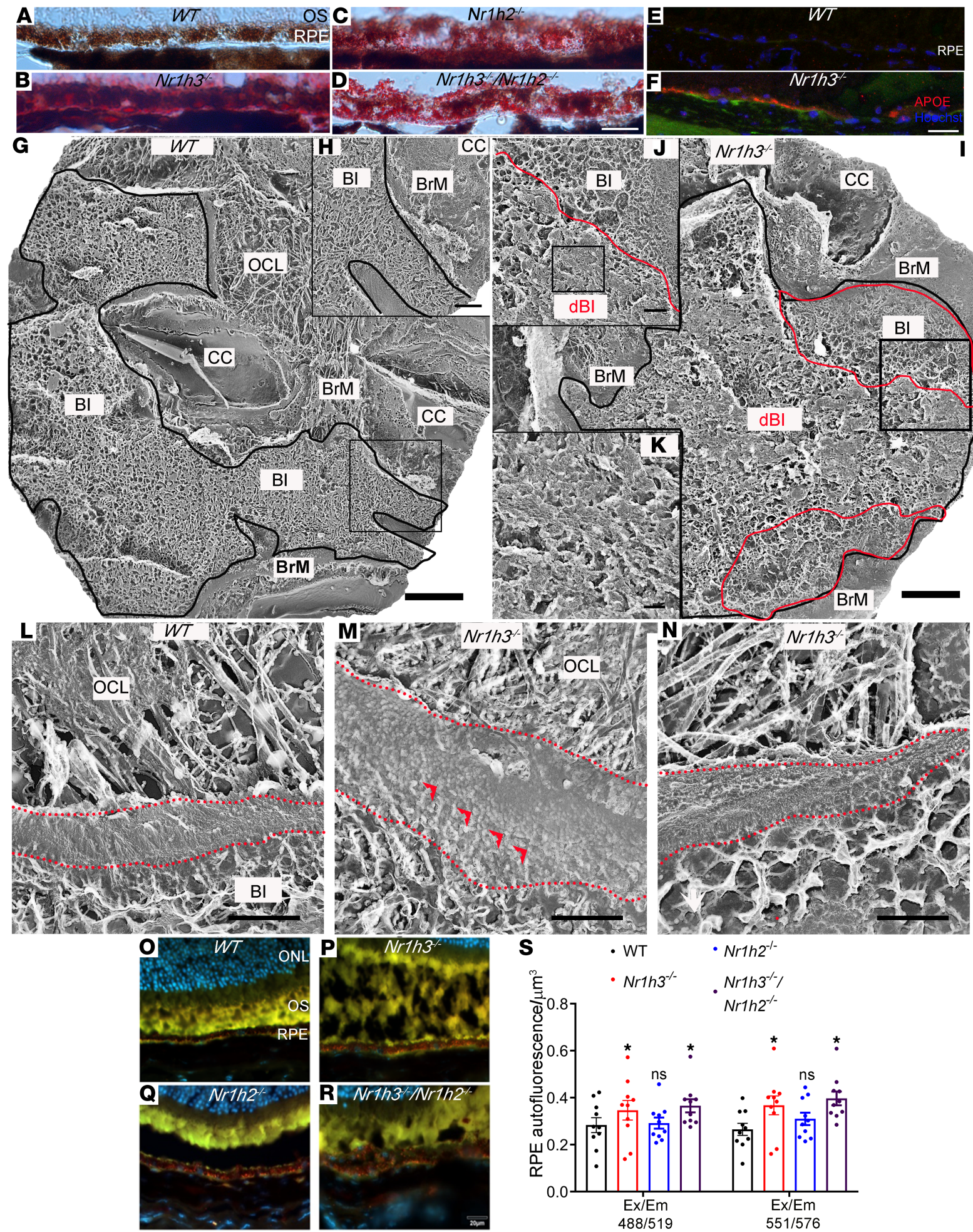

$\mathbf{s}$
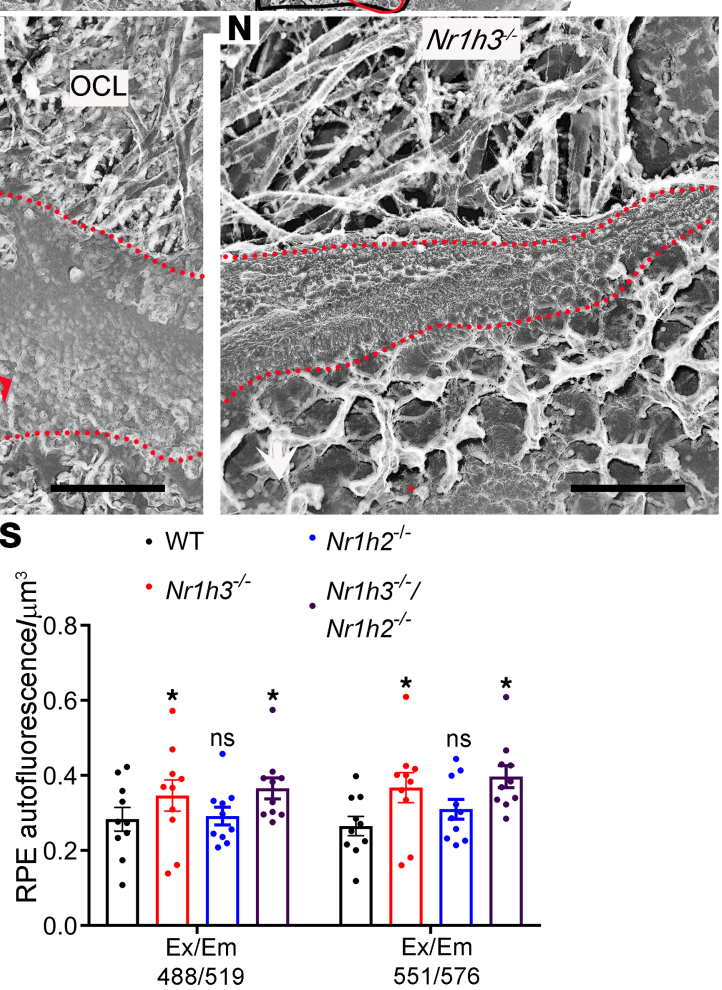

Figure 6. NR1H3 regulates lipid deposition in the outer retina. Representative images from retina/RPE/choroid sections of 10- to 14-month-old (A) WT ( $n$

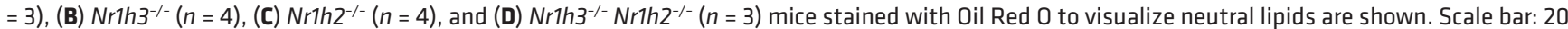
$\mu \mathrm{m}$. Retina/RPE/choroid sections from 10- to 14-month-old (E) WT $(n=3)(\mathbf{F}) \mathrm{Nr}_{\mathrm{h}} 3^{-/-}(n=4)$ mice were stained for APOE (red) and EO6 (green). Repre-

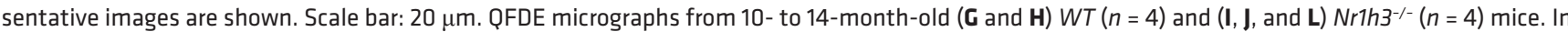
these micrographs, the fracture plan "hovers" between the RPE basal infoldings (BIs) and choriocapillaris (CC). (C) In the WT mouse, the Bls are located in

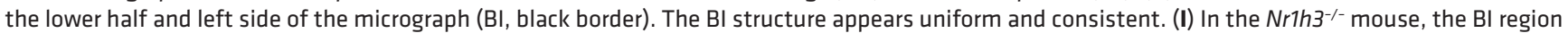
is located in the center of the micrograph and along the bottom edge (red border). Regions of the BI that have the unaffected structure (labeled $\mathrm{BI}$, within black border), are predominantly adjacent to $\mathrm{BrM}$, but most of the region in this micrograph shows a heavily disrupted $\mathrm{BI}$ (dBI). (H) Inset shows a higher magnification of a region that contains both healthy BI and BrM. (J) Inset shows the surface structure of the $\mathrm{dBI}$ and healthy BI. (K) Inset shows a high magnification of the dBI structure marked by the box in inset J. Scale bar: $4 \mu \mathrm{m}$ ( $\mathbf{G}$ and I), $1 \mu \mathrm{m}$ (inset $\mathbf{H}$ and J), $250 \mathrm{~nm}$ (inset $\mathbf{K}$ ). BrM, Bruch's membrane; 
OCL, outer collagenous layer. (L) Representative image of healthy BrM from WT mouse, red dotted line; scale bar: 500 nm. (M) Representative image from $\mathrm{Nr}_{\mathrm{h}} 3^{-/-}$mouse, showing spherical particles in BrM, red dotted line and red arrowheads; scale bar: $500 \mathrm{~nm}$. (N) A region of healthy BrM in the $\mathrm{Nr} 1 \mathrm{~h} 3^{-/-}$

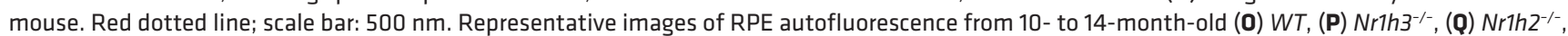

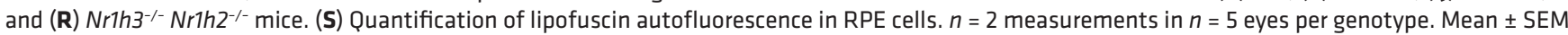
shown. ${ }^{*} P<0.05$, 1-way ANOVA, Dunnett's multiple-comparisons test.

Nr1h3 regulates lipid deposition in the outer retina. Accumulation of neutral lipids with age in BrM and extracellular to the RPE has been correlated with drusen formation $(29,30)$. Given the reported role of LXR in regulating lipid metabolism, we stained cryosections from $N r 1 h 3^{-/-}(n=4), N r 1 h 2^{-/-}(n=4), N r 1 h 3^{-1-}$ $N r 1 h 2^{-1-}(n=3)$, and $W T(n=3)$ mice with the histochemical stain oil red O (ORO) to evaluate the presence of neutral lipids within the retina. Although the RPE and BrM of WT mice were absent of neutral lipids (Figure 6A), $\mathrm{Nr} \mathrm{h}^{-/-}$mice displayed robust staining in sub-RPE deposits and within BrM (Figure 6B). $N r 1 h 2^{-/-}$mice displayed a strong globular staining pattern within and below the RPE cell layer (Figure 6C). $\mathrm{Nr}_{\mathrm{h}} \mathrm{3}^{-/-} \mathrm{Nr} 1 \mathrm{~h} 2^{-/-}$mice also displayed strong ORO-positive staining not only within the RPE cells but also in sub-RPE deposits (Figure 6D). ORO-positive staining in the tips of the photoreceptor outer segments, as seen in the WT panel, served as a positive internal control (Figure 6A). Another characteristic of sub-RPE deposits in AMD is the accumulation of APOE, a regulator of cholesterol transport, and oxidized lipoproteins $(31,32)$. Probing $\mathrm{Nr}_{1 h 3^{--}}(n=4)$ and $W T(n=3)$ eyes with antibodies targeting APOE and oxidized phospholipids present in oxidized LDL (E06) revealed higher levels of APOE and E06 immunoreactivity below the RPE in $\mathrm{Nr} 1 \mathrm{~h} 3^{-/-}$(Figure 6F) compared with WT sections (Figure 6E), suggesting the buildup of oxidized lipids along the length of BrM. QFDE imaging is a technique that allows for further interrogation of lipoprotein particles in human aged BrM (33-36). En face visualization with QFDE displayed severely disrupted basal infoldings of the RPE in $\mathrm{Nr} \mathrm{h}^{-/-}$(Figure 6, I-K, and Supplemental Figure 13), compared with uniform and organized basal infoldings in $W T$ mice (Figure 6, G and $\mathrm{H}$ ). A different angle and depth of fracture plane allowed us to examine the structure of the RPE basement membrane, inner collagenous layer, and elastic layer of BrM. Although the WT mice exhibited normal morphology, across BrM (Figure 6L), select regions within BrM of $\mathrm{Nr}_{\mathrm{h}} \mathrm{h}^{-/-}$mice presented with an accumulation of fused, round particles (Figure 6M and Supplemental Figure 13D). A region of the $N r 1 h 3^{-1-} \mathrm{BrM}$ that lacked the accumulation of these spherical particles is also shown (Figure $6 \mathrm{~N}$ ). Given that the CC is also compromised in dry AMD, we visualized the ultrastructure of the choriocapillary surface and found that in WT mice, fenestrations in

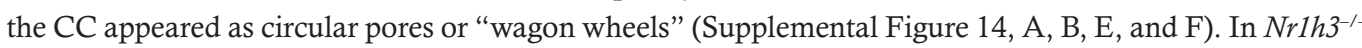
mice, fewer fenestrations were observed, and the "spokes" of the fenestrations were fewer in the "wagon wheels" (Supplemental Figure 14, C, D, G, and H). These results provide a unique representation of lipid deposition in regions below the RPE and details of changes in RPE morphology associated with the loss of Nr1h3. Finally, an evaluation of RPE autofluorescence revealed a similar isoform-dependent pattern of severity, with significant upregulation seen in $\mathrm{Nr}_{\mathrm{h}} \mathrm{h}^{-/-}$(16.7\%) and $\mathrm{Nr}_{\mathrm{h}} \mathrm{h}^{-/-} \mathrm{Nr} \mathrm{hh} \mathrm{2}^{-/-}$(20.0\%) compared with the $W T$ (Figure 6, O-S) mice and no significant changes seen in $N r 1 h 2^{-1-}$ RPE autofluorescence levels. These findings suggest that lipofuscin accumulation in the RPE cells, which may be a result of dysregulation of lipid metabolism and clearance, as well as accumulation of extracellular neutral lipid and apolipoprotein are at least in part attributable to $\mathrm{Nr} 1 \mathrm{~h} 3$.

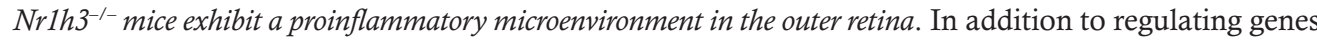
involved in cholesterol transport, LXR is involved in regulation of an array of proinflammatory genes resulting from insults such as LPS, TNF- $\alpha, \mathrm{IL}-1 \beta$, or bacterial stimulation (18), and it has been reported that LXR agonists may act as negative regulators of macrophage inflammatory gene expression (37). Of relevance, recruitment of immune cells to the outer retina is a purported feature of the AMD etiology (38). We examined the outer retina of $N r 1 h 3^{-/-}$mice for inflammatory mediators. RPE-choroid flat mounts were prepared from aged $W T(n=5)$ and $\operatorname{Nr}_{1 h 3^{--}}(n=8)$ mice, probed with a macrophage marker (ADGRE1, also known as F4/80), and stained with phalloidin to delineate the RPE cells. We observed a 3.8-fold accumulation of ADGRE1 ${ }^{+}$cells within the subretinal space of $N r 1 h 3^{-/}$compared with $W T$ mice (Figure 7, A-D), suggesting an augmented proinflammatory microenvironment in the subretinal space. However, we did not detect striking differences in the morphology of phalloidin-stained RPE cell borders (Supplemental Figure 15, A-C). To further evaluate the cytokine milieu of the outer retina, we examined the cytokine profile of the RPE-choroid tissue complex isolated from $W T$ and $N r 1 h 3^{-1-}$ mice. Out of a panel of 62 proteins, $N r 1 h 3^{-1-}$ samples exhibited a significant upregulation of 9 cytokines, with a primarily proinflammatory 

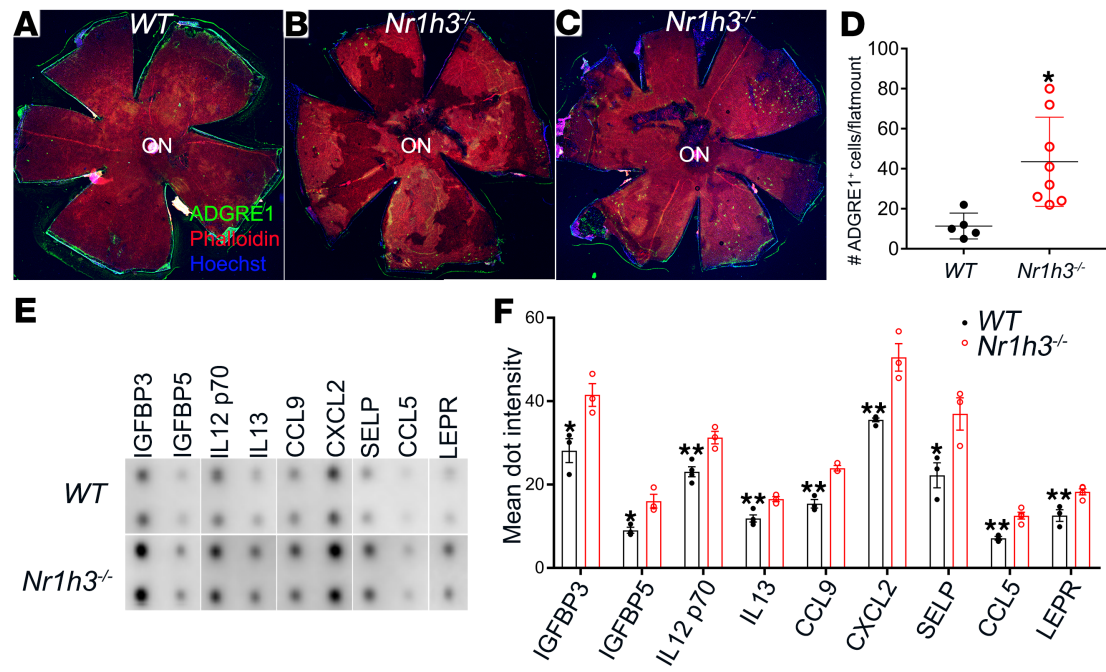

Figure 7. $\mathrm{Nr}^{1 \mathrm{h3}^{-/-}}$mice exhibit a proinflammatory microenvironment in the outer retina. RPE-choroid flat mounts from 10- to 14-month-old (A) WT and (B and C) $\mathrm{Nr}^{\mathrm{h} 3^{-1}}{ }^{-1}$. Two examples are shown. ON, optic nerve. Mice were stained with ADGRE1 (green) and phalloidin (red). Scale bar: $50 \mu \mathrm{m}$. (D) Quantification of ADGRE1 cells in RPE-choroid flat mounts from WT and $\mathrm{Nr} \mathrm{h} 3^{-/-}$mice. WT, $n=5 ; \mathrm{Nr} \mathrm{h} 3^{-/-}, n=8$. Mean $\pm \mathrm{SEM}$. ${ }^{*} P<0.01$. Unpaired $t$ test. (E) Protein isolated from RPE-choroid complexes of 10- to 14-month-old WT and $\mathrm{Nr}_{1} \mathrm{~h}^{-/-}$mice were blotted on a Mouse Cytokine Array C3 (62 proteins). Representative images of significantly regulated cytokines (2 dots/ protein) are shown. (F) Quantification of staining intensity of significantly regulated cytokines. $n=4$ per group. Mean \pm SEM shown. ${ }^{*} P<0.05 ;{ }^{*} P<0.01 ; 2$-tailed $t$ tests.

profile (Figure 7, E and F). Interestingly, at a systemic level, 10 cytokines were found to be downregulated in the plasma of $\mathrm{Nr}_{\mathrm{rh}} \mathrm{S}^{-/-}$mice (Supplemental Figure 16, A and B). These results corroborate that Nrlh3 is involved in maintaining a homeostatic microenvironment in the outer retina.

Therapeutic targeting of LXR diminishes pathogenic phenotypes associated with AMD. There is marked accumulation of neutral lipids in the pentalaminar BrM in a normal eye with aging $(39,40)$. This lipid buildup in the posterior eye has been theorized to set the stage for the formation of extracellular lipid-rich deposits and specifically the development of drusen and basal deposits, phenotypic characteristics of early dry AMD (39, 41, 42). Several studies have shown that this extracellular lipid deposition is, at least in part, due to the accumulation of esterified cholesterol-rich APOB-containing lipoprotein particles $(43,44)$. Because mice predominantly produce the apoB48 protein, we used a mouse model that produces the full-length mouse apoB100 (23), as a proof of concept, to evaluate the therapeutic effect of modulating LXR activity. It is well established that the RPE-choroid of young apoB100 mice produces the apoB100 isoform and there is accumulation of neutral lipids in their BrM (24). Herein, 3-month-old apoB100 mice were fed a low-fat diet (LFD) or the LXR agonist GW3965, supplemented in a low-fat diet (LFD + GW), for 5 months. Mice in both groups displayed a similar rate of increase in weight with age, suggesting absence of any overt systemic toxicities (Supplemental Figure 17A). We measured the expression of LXR target genes, Abca1 and Srebp1c, in RPE-choroid isolated from LFD- and LFD + GW-treated mice and found that their expression was upregulated in the LFD + GW cohort (Abca1: $54 \%$, Srebp1c: 35\%; $n=2$ ), indicating that the drug was able to reach the posterior retina and activate LXR locally. Additionally, no differences were observed in the systemic levels of HDL and LDL/VLDL (Supplemental Figure 17, B and C). Fundus and OCT imaging exhibited an improvement in the morphology of retinal layers as evident by a decrease in hypopigmented regions in fundus images of the LFD + GW group (Figure 8, C and D) as compared with LFD mice (Figure 8, A and B). We also observed a significant decrease in GFAP staining within the inner retina following activation of LXR in comparison with mice fed the LFD, supporting rescue of retinal injury associated with the apoB100 mice and a decline in inflammation (Figure 8, $\mathrm{E}$ and F). Quantification of the lipoprotein deposition in BrM of apoB100 mice revealed a significant decrease (77\%) in the fraction of $\mathrm{APOE}^{+}$length of BrM following LXR agonist treatment (Figure 8, G-I). GW3965 treatment also led to a reduction in the number of $\mathrm{ADGRE}^{+}$cells within the subretinal space $(81 \%, P=0.0598$; Figure 8, J-L). Furthermore, examination of the ultrastructure of the retina/RPE/choroid complex from vehicle-treated (LFD; Figure $8 \mathrm{M}$ ) and drug-treated mice (LFD + GW) revealed disrupted RPE basal infoldings (Figure $8 \mathrm{~N}$ ) and intermittent thin sub-RPE deposits (Figure 8O) in the LFD cohort. These morphological 

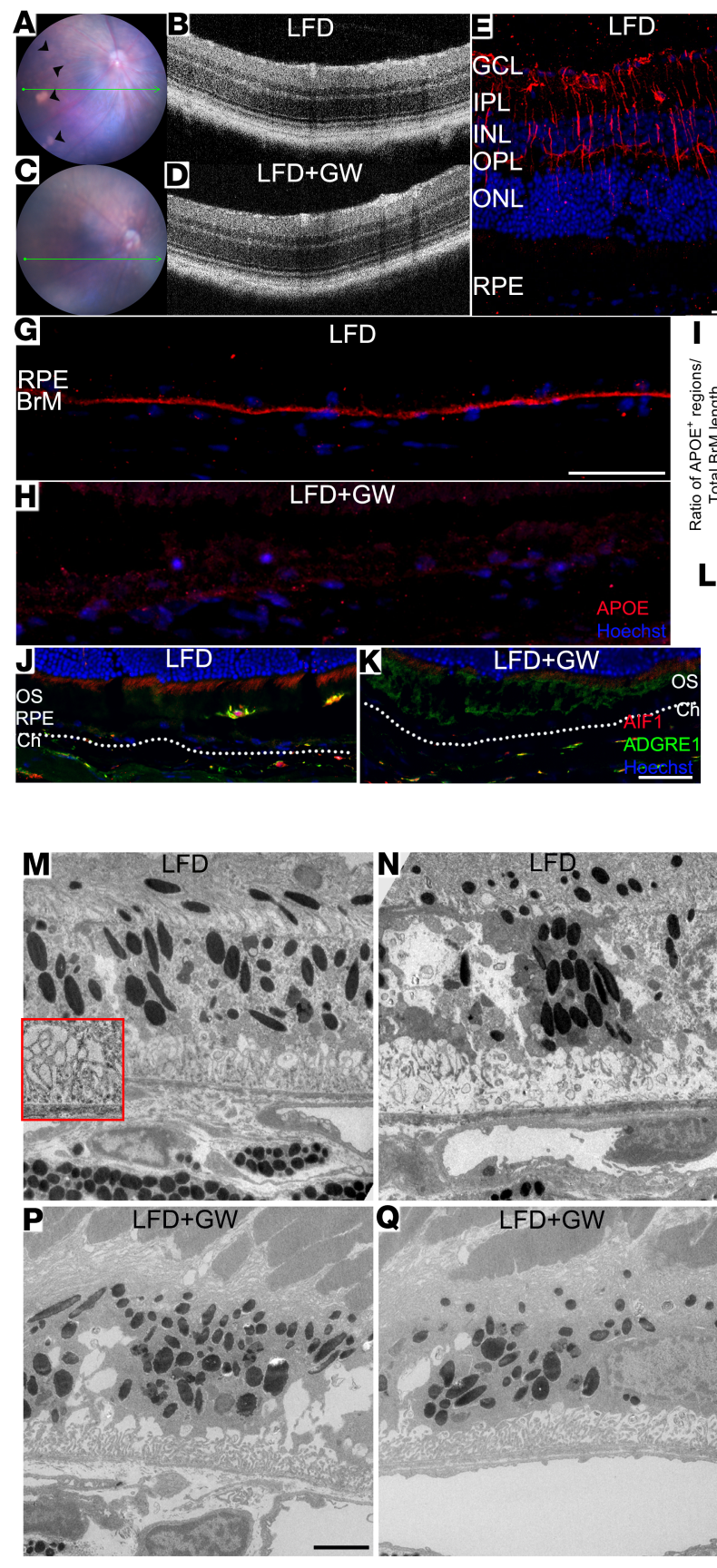
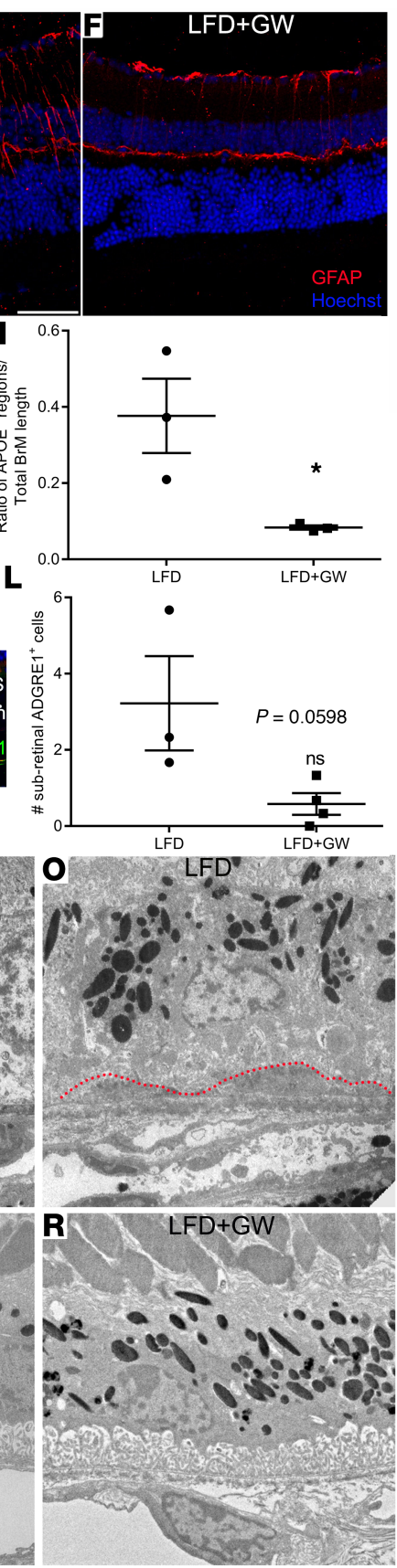

Figure 8. GW3965 diminishes pathogenic phenotypes associated with inflammation and lipid deposition. Fundus and OCT images of apoB100-expressing mice on control low-fat diet (LFD) diet (A and B) and LFD + CW3965 (GW) diet (C and D) after treatment. Black arrowheads point to hypopigmented regions in the fundus. GFAP staining in retina/RPE/choroid sections from LFD (E) and LFD + CW (F) mice. Representative images are shown. Scale bar: $50 \mu \mathrm{m}$. APOE immunolocalization in retina/RPE/choroid sections from LFD (G) and LFD + CW (H) mice. Representative images are shown. Scale bar: $50 \mu \mathrm{m}$. (I) Quantification of $\mathrm{APOE}^{+}$staining. $n$ $=3$ per group. Mean \pm SEM shown. ${ }^{*} P<0.05$, unpaired 2-tailed $t$ test. ADGRE1+ staining in retina/RPE/choroid sections from LFD (J) and LFD + CW (K) mice. Ch, choroid; dotted line depicts Bruch's membrane. Representative images are shown. Scale bar: $50 \mu \mathrm{m}$. (L) Quantification of subretinal ADGRE1+ cells. LFD, $n=3$. Mean \pm SEM shown. LFD $+\mathrm{CW}, n=$ 4. Ns, not significant, unpaired 2-tailed $t$ test. TEM images of the RPE cells from LFD-treated mice, showing (M) disrupted basal infoldings (red inset), (N) compromised RPE cells, and ( $\mathbf{O})$ sub-RPE deposits (red dotted line). (P-R) TEM images of the RPE cells from LFD + CW mice, showing improvement in RPE health, basal infoldings, and absence of subRPE deposits. Scale bar: $2 \mu \mathrm{m}$ (P).

features displayed a marked improvement in the LFD + GW group (Figure 8, P-R). Finally, the LFD + GW group displayed distinct mitochondria (Supplemental Figure 18, C and D) relative to the vehicle group (Supplemental Figure 18, A and B). These results signify that LXR activation is able to therapeutically influence inflammation and APOE deposition in the outer retina and may be a viable path for targeting these pathogenic pathways in AMD.

\section{Discussion}

The need to identify animal models that recapitulate early phenotypic features of complex neurodegenerative diseases, such as dry AMD, is a necessity to serve as a platform to not only further understand the pathobiology of disease but also facilitate testing of potential therapies in preclinical studies. We hypothesized that the LXR signaling pathway is likely an important mediator of AMD pathobiology given its role as a regulator of lipid metabolism, lipid transport, and inflammation, critical pathogenic pathways associated with disease development. To this end, we interrogated human ocular samples from donors with 
AMD for evidence of LXR involvement and found that the expression of LXR in human RPE cells, which are nurse cells to the retina and a primary vulnerable site in AMD, decreases as a function of age. Furthermore, we found that drusen, the characteristic lesion of early dry AMD, contain fragments that are LXR immunopositive. We demonstrated that the LXR signaling pathway can be activated in human RPE cells in vitro and characterized the ocular phenotype and function of Nr1h3-, Nr1h2-, and Nr1h3 Nr1h2-knockout mice. Interestingly, we found an isoform-dependent ocular phenotype, with $\mathrm{Nr} 1 \mathrm{h3^{-/ }}$ mice demonstrating accumulation of sub-RPE extracellular lipids, a distinguishing characteristic of early dry AMD, while the pathology of $\mathrm{Nr}_{\mathrm{h}} \mathrm{2}^{-/-}$mice mimicked RPE lipoidal degeneration. Preclinical studies allowed testing of the efficacy of an LXR agonist, selected based on its ability to reestablish RPE cholesterol homeostasis and mitochondrial function, in an in vivo model with phenotypic features of dry AMD. Collectively, our results have identified a potentially novel mouse model with characteristic features of dry AMD, identified mechanisms of LXR-based therapies, demonstrated efficacy of targeting the LXR pathway, and supported developing LXR-directed therapies for the treatment of neurodegenerative diseases of aging, such as AMD, in which altered cellular metabolism and inflammation may play a role.

This study is the first comprehensive characterization of the posterior ocular function and phenotype of LXR-knockout mice to our knowledge. Though redundancies have been shown in the function of the 2 LXR isoforms in other organs, including the liver, isoform-specific functions have also been discovered. Systemically, aged, LXR-deficient mice have been shown to display isoform-specific differences in plasma cholesterol levels, with absence of Nr1h2 associated with significant elevation of total cholesterol and HDL levels and absence of either Nr1h3 or Nr1h2 associated with increased LDL levels (45). Additionally, $N r 1 h 3^{-1-}$ mice have been shown to display rapid accumulation of large amounts of cholesterol, leading to impaired hepatic function (46). Conversely, $\mathrm{Nr} 1 \mathrm{h2^{-/- }}$ mice maintain their resistance to dietary cholesterol, despite differences in the expression of genes involved in lipid metabolism (47). Similarly, differences in expression of target genes have been reported in the epididymis of 12-month-old mice, with the absence of the $\beta$-isoform but not $\alpha$ associated with a significant loss of expression of the cholesterol transporters, Abcal and $A b c g 1$ (45). In our investigations, we found that the LXR pathway must also be critical for the development of normal visual function, because visual function deficits, mostly corresponding to the posterior region of the eye and specifically the RPE, were detected. Importantly, decrease in the function of RPE cells was observed primarily in mice deficient in Nr1h3. Furthermore, we found that both isoforms, along with their obligate binding partners, the RXRs, were expressed in several ocular tissues of the posterior pole, including the retina, RPE, and choroid. Isoform-specific variations were also found in physiological and pathological processes under the control of each of the isoforms in the eye. Absence of Nr1h2 affected the anterior segment, resulting in stellate cataract formation as well as accumulation of intracellular and extracellular lipid deposits, while Nr1h3-null mice primarily developed RPE extracellular lipid accumulation. When both isoforms were absent, not surprisingly, not only were all phenotypes evident, but also life expectancy was diminished with many mice perishing by 10-11 months of age.

That the LXR signaling pathway may be important in the aging eye is not surprising, given the importance of cholesterol regulation in the retinal milieu. The retina relies on both local synthesis as well as delivery from systemic circulation to replenish its cholesterol reserves $(48,49)$. Furthermore, it uses HDL-mediated reverse cholesterol transport and metabolism of cholesterol to more soluble oxysterols for its elimination $(49,50)$. The excess cholesterol may remain in the retina and undergo oxidation because of exposure to light and endogenous free radicals $(51,52)$. Given the fact that LXRs, key regulators of cholesterol homeostasis, are expressed in retina and RPE and oxysterols act as endogenous ligands for LXRs, it is plausible that LXRs may be one of the principal regulators of lipid metabolism and transport in the eye. This was confirmed by our in vivo assessments, which show accumulation of neutral and oxidized lipids below the RPE and within BrM, as evident by positive ORO and E06 staining in the absence of Nr1h3. Accumulation of oxidized lipids is an important contributor to AMD pathogenesis because these oxidized lipids can directly contribute to tissue injury or recruit inflammatory mediators in the outer retina (53). QFDE TEM is an imaging technique that has previously been used to visualize lipid particles, believed to be lipoproteins in human aged donor eyes (54). In conjunction with conventional TEM, we were able to study the topology and ultrastructure of lipid deposits in detail in the RPE/BrM complex. Although normal and healthy RPE basal infoldings in the $W T$ mice were seen, $N r 1 h 3^{-1-}$ mice displayed regions of severely disrupted basal infoldings amid healthy regions. The en face images of RPE/BrM/CC complex from $\mathrm{Nr}_{1 \mathrm{~h} 3^{-/-}}$mice acquired by this technique displayed "deposits" above the inner collagenous layer. 
Of note, the $\mathrm{Nr}_{\mathrm{hh}} \mathrm{H}^{-/-}$mice did not etch as well as the WT mice with QFDE, suggesting the presence of low volatiles, such as an abundance of lipids. This corroborates the histological and IHC findings in our mouse model of the presence of neutral lipids, using ORO staining, and apolipoproteins within BrM and below the RPE, pathologies characteristic of human AMD. This is the first demonstration to our knowledge of the pathology of the posterior pole of $\mathrm{Nr}_{\mathrm{h}} \mathrm{h}^{-/-}$mouse eye using QFDE and specifically visualization of disrupted basal infoldings and depots of spherical/lipid-like particles. Collectively our imaging results show that loss of $\mathrm{Nr} 1 \mathrm{~h} 3$ leads to accumulation of lipids in the RPE/BrM complex, which may stem from dysregulation of lipid clearance pathways in the eye.

Considerable evidence has emerged to indicate that the LXR pathway regulates inflammation and immune processes. In vivo, LXR-deficient mice have a sustained state of heightened proinflammatory mediators, with specifically $\mathrm{Nr}_{\mathrm{h}} \mathrm{h}^{-/-}$mice displaying exacerbated $\mathrm{CCl}_{4}$-induced lesions, in addition to inflammation and collagen deposition, in a model of chronic liver injury (55). In a model of retinal injury by $N$-methyl-D-aspartate, LXR activation reduced retinal neurotoxicity of the treatment and inhibited the increase of phospho-p38 MAPK and proinflammatory cytokine TNF- $\alpha$ in the treated mice (56). Additionally, activation of the LXR pathway represses a set of inflammatory genes, including Nos $2, m t-C o 2, I l 6$, and $I l 1 b$, after LPS, TNF- $\alpha$, or IL-1 $\beta$ stimulation (37). In our study, we have shown the accumulation of ADGRE $1^{+}$macrophages in the outer retina of $\mathrm{Nr}_{\mathrm{h}} \mathrm{h}^{-/-}$mice, indicating a proinflammatory microenvironment. We also measured the cytokine profile of the RPE-choroid complex and found a proinflammatory cytokine profile in $N r 1 h 3^{-1-}$ mice compared with the WT mice. This panel of cytokines included IGFBP3, IGFBP5, IL12 P70, IL13, CCL9, CXCL2, SELP, CCL5, and LEPR. IGF axis gene polymorphisms have been associated with advanced AMD, and specifically IGFBP3 and IGFBP5 have been shown to be expressed in RPE progressive phenotypes, namely normal, early reactive, and myofibroblasts $(57,58)$. IL-13 levels have also been found to be upregulated in the aqueous humor of patients diagnosed with neovascular AMD, suggesting a pathological role in AMD progression (59). CCL9 and CXCL2 have been reported to be involved in the recruitment of neutrophils to the site of injury and might act as a chemoattractant for the ADGRE1+ macrophages (60). Furthermore, CCR5 shows a trend to be elevated in a subset of monocytes isolated from treatment-naive neovascular AMD patients (61). Interestingly, we observed that systemic levels of cytokines significantly decreased in $\mathrm{Nr}_{1 \mathrm{~h} 3^{--}}$mice compared with the controls, some with reported protective properties. CXCL1 has been reported to confer a neuroprotective function in a model of experimental autoimmune encephalomyelitis (62). Colony stimulating factor 1 (CSF1) has been implicated in repair following acute kidney injury, suggesting that a decline in systemic CSF1 levels may have a detrimental effect on tissue homeostasis (63). The contrary trend seen in RPE-choroid and systemic levels of IGFBP3, CCL9, and CXCL2, together with the ocular phenotype observed in knockout mice, implies that local cytokine milieu may have a more dominant role in maintaining homeostasis and/or contributing to disease, especially in an organ such as the eye, which enjoys immune privilege. Though it remains to be seen if the inflammation observed in these mice is a direct consequence of the absence of $L X R$ or if it occurs in response to the elevated lipid accumulation, we construe that loss of $N r 1 h 3$ leads to a proinflammatory ocular environment, which may aid in the progression of the disease by altering RPE and/or choroidal endothelial cells.

Finding and developing therapies for dry AMD has been a challenge to date. This dilemma is, in part, due to the complexity of this neurodegenerative disease, with multiple retinal cells affected and pathogenic pathways involved, raising the question of which cells and/or pathways should be targeted. One strategy has been to target genetic risk factors or modify environmental factors associated with disease progression. An alternative strategy would be to strengthen the armor of AMD-vulnerable cells and reinforce pathways that are compromised as a function of age. We found that expression of $L X R$ s decreases as a function of age and, in particular, in the "elderly" risk age group, supporting the hypothesis that activating LXR may have beneficial effects on the overall health of RPE cells. Two factors must be considered in pursuing preclinical testing of an LXR-targeted therapy.

The first is the choice of activating ligand. To date, several activating LXR ligands have been reported to modulate lipid metabolism and inflammatory pathways in different injury models, including the eye. Activating LXR with GW3965 has been shown to provide a protective effect in experimental diabetic retinopathy and an inner retinal damage mouse model $(56,64)$, and the LXR agonist TO901317 has been shown to ameliorate amyloid $\beta$-induced inflammatory responses in human RPE cells as well as the neural retina (65, 66). It is important to note, however, that LXR ligands are not without side effects, most notably temporary hypertriglyceridemia. Furthermore, the TO901317 compound, the first LXR ligand to be identified and one that has been tested frequently by researchers, has potential off-target effects through activation of other 
nuclear receptors, including farnesoid X receptor and pregnane X receptor $(67,68)$. The selectivity of LXR ligands can be attributed to several factors, including degree of induction of genes involved in lipid transport, namely, $A B C A 1, A B G A 1$, and $A P O E$, or of transrepression of proinflammatory genes, such as nitric oxide synthase and cyclooxygenase-2, or proinflammatory cytokines in microglia and astrocytes $(47,69,70)$. It has further been proposed, but unproved, that GW3965 and TO901317 may bind to both isoforms with varying degrees of binding affinity (71). In our studies, in vitro assays allowed comparison of the function of agonists GW3965 and TO901317, versus the antagonist GSK2033 in RPE and choroidal endothelial cells. We found these activating ligands bind to both isoforms, NR1H3 and NR1H2, as evident by the induction of promoter activity and target gene expression, in the presence of siRNA targeting individual isoforms. Our in vitro, AMD-relevant endpoints for assessing LXR ligand efficacy included mitochondrial membrane potential, as a measure of mitochondrial health, and lipid accumulation. The choice to assess the mitochondria was in light of the extensive evidence supporting a contributory role of mitochondrial dysfunction, changes in mitochondrial number and size, increased mitochondrial DNA damage, and a decline in DNA damage repair in AMD (72-75). Furthermore, the mitochondrial enzyme cytochrome P450 27A1 (CYP27A1) regulates cholesterol homeostasis, bile acid biosynthesis, activation of vitamin $\mathrm{D}_{3}$, and removal of the cytotoxic metabolite 7-ketocholesterol, and its absence in mice results in upregulation of cholesterol biosynthesis in the mouse retina, along with sub-RPE accumulation $(76,77)$, supporting additional contributions of mitochondrial function to AMD pathogenesis. We found that the ability of LXR activation to prevent mitochondrial injury when RPE cells are exposed to multiple oxidative insults, including treatment with sodium iodate, hydrogen peroxide, CSE, and lipid overload, was selective and based on the type of oxidant injury. TO901317 was effective in countering "classical" oxidant injury, such as $\mathrm{NaIO}_{3}, \mathrm{H}_{2} \mathrm{O}_{2}$, and CSE, whereas GW3965 was effective in ameliorating mitochondrial injury induced by lipid treatment. Similarly, we found that only GW3965 was able to prevent intracellular lipid accumulation in response to treatment with PA and $\alpha$-LA in RPE cells.

The second factor to be considered for preclinical studies is the choice of animal model. We tested the therapeutic effect of LXR activation on lipid deposition and inflammation in the apoB100 mouse model, which is characterized by accumulation of neutral lipids below the RPE (24), a phenotypic feature of aging and potential precursor to development of AMD. Furthermore, in these mice, the cholesterol transporter, apoE, also accumulates below the RPE in the space where sub-RPE deposits develop (32). We chose to deliver the drug via diet because systemic delivery of LXR ligands has been used to target the brain and both GW3965 and TO901317 have been shown to pass the blood-brain barrier $(78,79)$. We observed that apoB100 mice fed a diet rich in GW3965 for 5 months displayed a decline in expression of inflammatory markers (GFAP and ADGRE1) within the retina and apoE deposition within BrM, supporting the therapeutic potential of activating LXR to target these AMD-associated pathologies.

Several studies to date have examined the phenotype of tissue-specific knockdown of downstream target genes of the LXR, including $A B C A 1$ in photoreceptor and RPE cells $(80,81)$. In the absence of $A b c a 1$ in mouse photoreceptors, there is accumulation of neutral lipid-rich subretinal debris and Aif $1^{+}$subretinal cells, along with compromise in visual function (81) while the absence of $A b c a 1$ in the mouse RPE demonstrated retinal degenerative changes, including significant RPE and retinal degeneration concomitant with RPE lipoidal degeneration, a phenotype also seen in our $\mathrm{Nr}_{\mathrm{H}} \mathrm{h}^{-1-}$ mice (80). Given previous findings that Abcal levels are affected more so in the absence of the $\beta$-isoform than $\alpha$-isoform, it is plausible that downstream effectors other than Abcal may be involved in the accumulation of lipids extracellular to RPE cells (e.g., the lipid-rich drusen phenotype). Additionally, phenotypic characterization of $N r 1 h 2^{-1-}$ mice, which exhibit inflammation of the optic nerve and loss of ganglion cells with aging, suggests that the $\beta$-isoform is a major contributor to the regulation of inflammation in the inner retina (82). Ban et al. targeted the lipid efflux pathway in macrophages by deleting Abcal and Abcg1 and found that the double-knockout mice present with extracellular lipid-rich deposits in the outer retina, below the retina, concomitant with photoreceptor dysfunction (83). Collectively, these reports in conjunction with our results support that both LXR isoforms are involved in the regulation of inflammation and lipid deposition in the inner and outer retina, but in an isoform-specific manner, and support the need for future studies examining the impact of LXR using tissue-specific knockout models.

Overall, our study establishes the LXR pathway as a key regulator of lipid transport and inflammation in the eye. Aged mice lacking the $\alpha$-isoform represent a new mouse model of the dry AMD phenotype and may be used as a platform to test therapies specifically targeting excess extracellular lipid accumulation and inflammation. Further, we identified mechanisms of LXR-based therapies and demonstrated in vivo 
efficacy of targeting the LXR pathway. Importantly, our results underscore the importance of the health of RPE cells, located at an important interface to regulate delivery of cholesterol to the retina as well as its elimination, highlighting their importance as a target for ocular pathologies governed by excessive lipid accumulation in the posterior pole (84). Broadly, this translational study supports developing LXR-targeted therapies for the treatment of neurodegenerative diseases of aging, in which altered cellular metabolism and chronic inflammation may play a role.

\section{Methods}

Human tissue and cell lines. Use of human donor eyes for research was approved by the Duke University Institutional Review Board. Details of methods associated with tissue collection and cell culturing are provided in the supplemental materials.

Animals. Male and female $N r 1 h 3 N r 1 h 2^{+/+}(W T), N r 1 h 3^{-/-}, N r 1 h 2^{-/-}$, and $N r 1 h 3^{--_{-}} N r 1 h 2^{-/-}$mice on the C57BL/6J background were used to examine the effect of the absence of $N r 1 h 2$ and $N r 1 h 3$ in vivo. Mice that express apoB100 (apoB100 mice, genetic background: 50\% C57BL/6 and 50\% 129/Sv) (24) were used to evaluate the effect of an LXR agonist in vivo. Additional details are provided in the supplemental materials.

Supplemental methods. Additional details of methods, including RNA isolation, quantitative PCR, siRNA transfection, transcriptional activity, AdipoRed, cell viability assays, JC-1 assay, blood collection, cytokine array, and postmortem tissue analyses, are provided in the Supplemental Methods and Supplemental Table 5.

Statistics. Statistical methods for data analysis included 2-tailed $t$ test and 2-way ANOVA, with Tukey's/ Dunnett's (as recommended by GraphPad Prism) multiple-comparisons test using GraphPad Prism. Values were considered statistically significant at $P<0.05$ and are indicated in the figures. For in vitro experiments, including transcriptional activity assays, JC-1, AdipoRed, and cell viability, samples were run in triplicate, and experiments were performed a minimum of 3 times (technical and biological replicates $=3$ ).

Study approval. The study protocols were approved by the Duke University Institutional Animal Care and Use Committees, and all animal experiments were performed in accordance with the guidelines of the Association for Research in Vision and Ophthalmology Statement for the Use of Animals in Ophthalmic and Vision Research.

\section{Author contributions}

MC, PLY, and FT participated in the collection of samples, data, or data analysis. MEB performed autofluorescence measurements. ENI and JWR performed QFDE. PT provided $L X R$-knockout mice. RAR, $\mathrm{SN}$, and RSA assisted in visual function testing, ERG analysis, or Fundus-OCT imaging. JTH provided apoB100 mice. MC and GM wrote the manuscript. GM edited the manuscript. All authors have read and approved the manuscript for publication.

\section{Acknowledgments}

This research was supported by the National Eye Institute grants R01 EY027802 (GM), R01 EY028160 (GM), P30 EY005722 (to the Duke Eye Center), EY000331 (to the Stein Eye Institute, Core Grant for Vision Research), R01 EY019287-06 (RSA), P30 EY02687 (to Washington University, Core Grant for Vision Research), and R01 EY027691 (JTH, Robert Bond Welch Professor); the Edward N. \& Della L. Thome Memorial Foundation Award (GM); the Carl and Mildred Almen Reeves Foundation (RSA); the Starr Foundation (RSA); the Bill and Emily Kuzma Family Gift for Retinal Research (RSA); the Jeffrey Fort Innovation Fund (RSA); the Glenn Foundation for Medical Research and the Thome Foundation (RSA); and the Research to Prevent Blindness, Inc, Core grant (to the Duke Eye Center). We are grateful to the donors and donor families for their generosity. We thank Neal Peachy for assistance with the c-wave recording protocols. Sincere thanks to Donald P. McDonnell and Ching-Yi Chang for plasmids used in transcriptional activity assays and valuable discussions. We thank Abdoulaye Sene, Xiaoping Qi, Eddie Meade, and Michael Lekwuwa for technical support.

Address correspondence to: Goldis Malek, Department of Ophthalmology, Duke University School of Medicine, 2351 Erwin Road, PO Box 3802, Room 4006, Durham, North Carolina 27710, USA. Phone: 919.684.0820; Email: gmalek@duke.edu. 
1. Miller JW, Bagheri S, Vavvas DG. Advances in age-related macular degeneration understanding and therapy. US Ophthalmic Rev. 2017;10(2):119-130

2. Wong WL, et al. Global prevalence of age-related macular degeneration and disease burden projection for 2020 and 2040: a systematic review and meta-analysis. Lancet Glob Health. 2014;2(2):e106-e116.

3. Mullins RF, Johnson MN, Faidley EA, Skeie JM, Huang J. Choriocapillaris vascular dropout related to density of drusen in human eyes with early age-related macular degeneration. Invest Ophthalmol Vis Sci. 2011;52(3):1606-1612.

4. Curcio CA, Presley JB, Millican CL, Medeiros NE. Basal deposits and drusen in eyes with age-related maculopathy: evidence for solid lipid particles. Exp Eye Res. 2005;80(6):761-775.

5. Sarks JP, Sarks SH, Killingsworth MC. Evolution of soft drusen in age-related macular degeneration. Eye (Lond). 1994;8 (Pt 3):269-283

6. Thornton J, Edwards R, Mitchell P, Harrison RA, Buchan I, Kelly SP. Smoking and age-related macular degeneration: a review of association. Eye (Lond). 2005;19(9):935-944.

7. Baird PN, Guida E, Chu DT, Vu HT, Guymer RH. The epsilon2 and epsilon4 alleles of the apolipoprotein gene are associated with age-related macular degeneration. Invest Ophthalmol Vis Sci. 2004;45(5):1311-1315.

8. Haines JL, et al. Complement factor $\mathrm{H}$ variant increases the risk of age-related macular degeneration. Science. 2005;308(5720):419-421.

9. Fritsche LG, et al. A large genome-wide association study of age-related macular degeneration highlights contributions of rare and common variants. Nat Genet. 2016;48(2):134-143.

10. Sonoda J, Pei L, Evans RM. Nuclear receptors: decoding metabolic disease. FEBS Lett. 2008;582(1):2-9.

11. Pardee K, Reinking J, Krause H. Nuclear hormone receptors, metabolism, and aging: what goes around comes around. Transcription factors link lipid metabolism and aging-related processes. Sci Aging Knowledge Environ. 2004;2004(47):re8

12. Moutinho M, Landreth GE. Therapeutic potential of nuclear receptor agonists in Alzheimer's disease. J Lipid Res. 2017;58(10):1937-1949

13. Skerrett R, Malm T, Landreth G. Nuclear receptors in neurodegenerative diseases. Neurobiol Dis. 2014;72(Pt A):104-116.

14. Ma Z, et al. Liver X Receptors and their agonists: targeting for cholesterol homeostasis and cardiovascular diseases. Curr Issues Mol Biol. 2017;22:41-64.

15. Dhiman VK, Bolt MJ, White KP. Nuclear receptors in cancer - uncovering new and evolving roles through genomic analysis. Nat Rev Genet. 2018;19(3):160-174.

16. Dwyer MA, Kazmin D, Hu P, McDonnell DP, Malek G. Research resource: nuclear receptor atlas of human retinal pigment epithelial cells: potential relevance to age-related macular degeneration. Mol Endocrinol. 2011;25(2):360-372.

17. Calkin AC, Tontonoz P. Liver x receptor signaling pathways and atherosclerosis. Arterioscler Thromb Vasc Biol. 2010;30(8):1513-1518.

18. Zelcer N, Tontonoz P. Liver X receptors as integrators of metabolic and inflammatory signaling. J Clin Invest. 2006;116(3):607-614

19. Malek G, Lad EM. Emerging roles for nuclear receptors in the pathogenesis of age-related macular degeneration. Cell Mol Life Sci. 2014;71(23):4617-4636

20. Chen W, et al. Genetic variants near TIMP3 and high-density lipoprotein-associated loci influence susceptibility to age-related macular degeneration. Proc Natl Acad Sci USA. 2010;107(16):7401-7406.

21. Neale BM, et al. Genome-wide association study of advanced age-related macular degeneration identifies a role of the hepatic lipase gene (LIPC). Proc Natl Acad Sci USA. 2010;107(16):7395-7400.

22. Edwards PA, Kennedy MA, Mak PA. LXRs; oxysterol-activated nuclear receptors that regulate genes controlling lipid homeostasis. Vascul Pharmacol. 2002;38(4):249-256.

23. Farese RV, et al. Phenotypic analysis of mice expressing exclusively apolipoprotein B48 or apolipoprotein B100. Proc Natl Acad Sci USA. 1996;93(13):6393-6398.

24. Fujihara M, Cano M, Handa JT. Mice that produce ApoB100 lipoproteins in the RPE do not develop drusen yet are still a valuable experimental system. Invest Ophthalmol Vis Sci. 2014;55(11):7285-7295.

25. Beatty S, Koh H, Phil M, Henson D, Boulton M. The role of oxidative stress in the pathogenesis of age-related macular degeneration. Surv Ophthalmol. 2000;45(2):115-134.

26. Pinto LH, Invergo B, Shimomura K, Takahashi JS, Troy JB. Interpretation of the mouse electroretinogram. Doc Ophthalmol. 2007;115(3):127-136.

27. Fine BS. Lipoidal degeneration of the retinal pigment epithelium. Am J Ophthalmol. 1981;91(4):469-473.

28. Rudolf M, et al. Apolipoprotein A-I mimetic peptide L-4F removes Bruch's membrane lipids in aged nonhuman primates. Invest Ophthalmol Vis Sci. 2019;60(2):461-472.

29. Curcio CA, Johnson M, Huang JD, Rudolf M. Aging, age-related macular degeneration, and the response-to-retention of apolipoprotein B-containing lipoproteins. Prog Retin Eye Res. 2009;28(6):393-422.

30. Ebrahimi KB, Handa JT. Lipids, lipoproteins, and age-related macular degeneration. J Lipids. 2011;2011:802059.

31. Klaver CC, et al. Genetic association of apolipoprotein E with age-related macular degeneration. Am J Hum Genet. 1998;63(1):200-206.

32. Anderson $\mathrm{DH}$, et al. Local cellular sources of apolipoprotein $\mathrm{E}$ in the human retina and retinal pigmented epithelium: implications for the process of drusen formation. Am J Ophthalmol. 2001;131(6):767-781.

33. Heuser J. Preparing biological samples for stereomicroscopy by the quick-freeze, deep-etch, rotary-replication technique. Methods Cell Biol. 1981;22:97-122.

34. Ismail EN, Ruberti JW, Malek G. Quick-freeze/deep-etch electron microscopy visualization of the mouse posterior pole. Exp Eye Res. 2017;162:62-72.

35. Ruberti JW, Curcio CA, Millican CL, Menco BP, Huang JD, Johnson M. Quick-freeze/deep-etch visualization of age-related lipid accumulation in Bruch's membrane. Invest Ophthalmol Vis Sci. 2003;44(4):1753-1759.

36. Huang JD, Presley JB, Chimento MF, Curcio CA, Johnson M. Age-related changes in human macular Bruch's membrane as seen by quick-freeze/deep-etch. Exp Eye Res. 2007;85(2):202-218.

37. Joseph SB, Castrillo A, Laffitte BA, Mangelsdorf DJ, Tontonoz P. Reciprocal regulation of inflammation and lipid metabolism by liver X receptors. Nat Med. 2003;9(2):213-219. 
38. Ambati J, Atkinson JP, Gelfand BD. Immunology of age-related macular degeneration. Nat Rev Immunol. 2013;13(6):438-451.

39. Curcio CA, Millican CL, Bailey T, Kruth HS. Accumulation of cholesterol with age in human Bruch's membrane. Invest Ophthalmol Vis Sci. 2001;42(1):265-274.

40. Rudolf M, Curcio CA. Esterified cholesterol is highly localized to Bruch's membrane, as revealed by lipid histochemistry in wholemounts of human choroid. J Histochem Cytochem. 2009;57(8):731-739.

41. Curcio CA, Johnson M, Rudolf M, Huang JD. The oil spill in ageing Bruch membrane. Br J Ophthalmol. 2011;95(12):1638-1645.

42. Li CM, et al. Lipoprotein-like particles and cholesteryl esters in human Bruch's membrane: initial characterization. Invest Ophthalmol Vis Sci. 2005;46(7):2576-2586.

43. Malek G, Li CM, Guidry C, Medeiros NE, Curcio CA. Apolipoprotein B in cholesterol-containing drusen and basal deposits of human eyes with age-related maculopathy. Am J Pathol. 2003;162(2):413-425.

44. Curcio CA, Johnson M, Huang JD, Rudolf M. Apolipoprotein B-containing lipoproteins in retinal aging and age-related macular degeneration. J Lipid Res. 2010;51(3):451-467.

45. Whitfield M, et al. Liver X receptors (LXRs) alpha and beta play distinct roles in the mouse epididymis. Biol Reprod. 2016;94(3):55.

46. Peet DJ, et al. Cholesterol and bile acid metabolism are impaired in mice lacking the nuclear oxysterol receptor LXR alpha. Cell. 1998;93(5):693-704.

47. Alberti S, et al. Hepatic cholesterol metabolism and resistance to dietary cholesterol in LXRbeta-deficient mice. J Clin Invest. 2001;107(5):565-573.

48. Fliesler SJ, Florman R, Rapp LM, Pittler SJ, Keller RK. In vivo biosynthesis of cholesterol in the rat retina. FEBS Lett. 1993;335(2):234-238.

49. Pikuleva IA, Curcio CA. Cholesterol in the retina: the best is yet to come. Prog Retin Eye Res. 2014;41:64-89.

50. Mast N, et al. Cholestenoic Acid is an important elimination product of cholesterol in the retina: comparison of retinal cholesterol metabolism with that in the brain. Invest Ophthalmol Vis Sci. 2011;52(1):594-603.

51. Saadane A, Mast N, Dao T, Ahmad B, Pikuleva IA. Retinal hypercholesterolemia triggers cholesterol accumulation and esterification in photoreceptor cells. J Biol Chem. 2016;291(39):20427-20439.

52. Bretillon L, et al. Lipid and fatty acid profile of the retina, retinal pigment epithelium/choroid, and the lacrimal gland, and associations with adipose tissue fatty acids in human subjects. Exp Eye Res. 2008;87(6):521-528.

53. Handa JT, Cano M, Wang L, Datta S, Liu T. Lipids, oxidized lipids, oxidation-specific epitopes, and Age-related Macular Degeneration. Biochim Biophys Acta Mol Cell Biol Lipids. 2017;1862(4):430-440.

54. Johnson M, Dabholkar A, Huang JD, Presley JB, Chimento MF, Curcio CA. Comparison of morphology of human macular and peripheral Bruch's membrane in older eyes. Curr Eye Res. 2007;32(9):791-799.

55. Xing Y, Zhao T, Gao X, Wu Y. Liver X receptor $\alpha$ is essential for the capillarization of liver sinusoidal endothelial cells in liver injury. Sci Rep. 2016;6:21309.

56. Zheng S, Yang H, Chen Z, Zheng C, Lei C, Lei B. Activation of liver X receptor protects inner retinal damage induced by N-methyl-D-aspartate. Invest Ophthalmol Vis Sci. 2015;56(2):1168-1180.

57. Chiu CJ, et al. Associations between genetic polymorphisms of insulin-like growth factor axis genes and risk for age-related macular degeneration. Invest Ophthalmol Vis Sci. 2011;52(12):9099-9107.

58. Mukherjee S, King JL, Guidry C. Phenotype-associated changes in retinal pigment epithelial cell expression of insulin-like growth factor binding proteins. Invest Ophthalmol Vis Sci. 2009;50(11):5449-5455.

59. Fu B, Liu ZL, Zhang H, Gu F. Interleukin-13 and age-related macular degeneration. Int J Ophthalmol. 2017;10(4):535-540.

60. Kobayashi Y. The role of chemokines in neutrophil biology. Front Biosci. 2008;13:2400-2407.

61. Grunin M, Burstyn-Cohen T, Hagbi-Levi S, Peled A, Chowers I. Chemokine receptor expression in peripheral blood monocytes from patients with neovascular age-related macular degeneration. Invest Ophthalmol Vis Sci. 2012;53(9):5292-5300.

62. Omari KM, Lutz SE, Santambrogio L, Lira SA, Raine CS. Neuroprotection and remyelination after autoimmune demyelination in mice that inducibly overexpress CXCL1. Am J Pathol. 2009;174(1):164-176.

63. Zhang MZ, et al. CSF-1 signaling mediates recovery from acute kidney injury. J Clin Invest. 2012;122(12):4519-4532.

64. Hazra S, et al. Liver X receptor modulates diabetic retinopathy outcome in a mouse model of streptozotocin-induced diabetes. Diabetes. 2012;61(12):3270-3279.

65. Lei C, et al. Amelioration of amyloid $\beta$-induced retinal inflammatory responses by a LXR agonist TO901317 is associated with inhibition of the NF-кB signaling and NLRP3 inflammasome. Neuroscience. 2017;360:48-60.

66. Dai B, et al. Activation of liver $\mathrm{X}$ receptor $\alpha$ protects amyloid $\beta_{1-40}$ induced inflammatory and senescent responses in human retinal pigment epithelial cells. Inflamm Res. 2017;66(6):523-534.

67. Houck KA, et al. T0901317 is a dual LXR/FXR agonist. Mol Genet Metab. 2004;83(1-2):184-187.

68. Mitro N, Vargas L, Romeo R, Koder A, Saez E. T0901317 is a potent PXR ligand: implications for the biology ascribed to LXR. FEBS Lett. 2007;581(9):1721-1726.

69. Paterniti I, et al. Liver X receptor agonist treatment regulates inflammatory response after spinal cord trauma. J Neurochem. 2010;112(3):611-624.

70. Xu P, et al. LXR agonists: new potential therapeutic drug for neurodegenerative diseases. Mol Neurobiol. 2013;48(3):715-728

71. Komati R, Spadoni D, Zheng S, Sridhar J, Riley KE, Wang G. Ligands of therapeutic utility for the liver X receptors. Molecules. 2017;22(1):E88.

72. Jarrett SG, Lin H, Godley BF, Boulton ME. Mitochondrial DNA damage and its potential role in retinal degeneration. Prog Retin Eye Res. 2008;27(6):596-607.

73. Feher J, Kovacs I, Artico M, Cavallotti C, Papale A, Balacco Gabrieli C. Mitochondrial alterations of retinal pigment epithelium in age-related macular degeneration. Neurobiol Aging. 2006;27(7):983-993.

74. Karunadharma PP, Nordgaard CL, Olsen TW, Ferrington DA. Mitochondrial DNA damage as a potential mechanism for age-related macular degeneration. Invest Ophthalmol Vis Sci. 2010;51(11):5470-5479.

75. Lin H, et al. Mitochondrial DNA damage and repair in RPE associated with aging and age-related macular degeneration. Invest Ophthalmol Vis Sci. 2011;52(6):3521-3529. 
76. Liao WL, et al. Quantification of cholesterol-metabolizing P450s CYP27A1 and CYP46A1 in neural tissues reveals a lack of enzyme-product correlations in human retina but not human brain. J Proteome Res. 2011;10(1):241-248.

77. Omarova S, et al. Abnormal vascularization in mouse retina with dysregulated retinal cholesterol homeostasis. J Clin Invest. 2012;122(8):3012-3023.

78. Liang Y, et al. A liver X receptor and retinoid X receptor heterodimer mediates apolipoprotein E expression, secretion and cholesterol homeostasis in astrocytes. J Neurochem. 2004;88(3):623-634

79. Namjoshi DR, et al. The liver X receptor agonist GW3965 improves recovery from mild repetitive traumatic brain injury in mice partly through apolipoprotein E. PLOS ONE. 2013;8(1):e53529.

80. Storti F, et al. Impaired ABCA1/ABCG1-mediated lipid efflux in the mouse retinal pigment epithelium (RPE) leads to retinal degeneration. Elife. 2019;8:e45100.

81. Ban N, et al. Disrupted cholesterol metabolism promotes age-related photoreceptor neurodegeneration. J Lipid Res. 2018;59(8):1414-1423.

82. Song XY, et al. Retinal and optic nerve degeneration in liver X receptor $\beta$ knockout mice. Proc Natl Acad Sci USA. 2019;116(33):16507-16512.

83. Ban $\mathrm{N}$, et al. Impaired monocyte cholesterol clearance initiates age-related retinal degeneration and vision loss. JCI Insight 2018;3(17):120824.

84. Fliesler SJ, Bretillon L. The ins and outs of cholesterol in the vertebrate retina. J Lipid Res. 2010;51(12):3399-3413. 